\title{
Finite similitude in fracture mechanics
}

DOI:

10.1016/j.engfracmech.2021.107573

\section{Document Version}

Accepted author manuscript

Link to publication record in Manchester Research Explorer

\section{Citation for published version (APA):}

Davey, K., Darvizeh, R., \& Zhang, J. (2021). Finite similitude in fracture mechanics. Engineering Fracture Mechanics, 245, [107573]. https://doi.org/10.1016/j.engfracmech.2021.107573

\section{Published in:}

Engineering Fracture Mechanics

\section{Citing this paper}

Please note that where the full-text provided on Manchester Research Explorer is the Author Accepted Manuscript or Proof version this may differ from the final Published version. If citing, it is advised that you check and use the publisher's definitive version.

\section{General rights}

Copyright and moral rights for the publications made accessible in the Research Explorer are retained by the authors and/or other copyright owners and it is a condition of accessing publications that users recognise and abide by the legal requirements associated with these rights.

\section{Takedown policy}

If you believe that this document breaches copyright please refer to the University of Manchester's Takedown Procedures [http://man.ac.uk/04Y6Bo] or contact uml.scholarlycommunications@manchester.ac.uk providing relevant details, so we can investigate your claim.

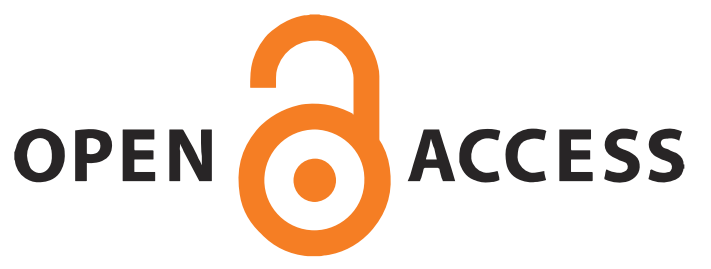




\title{
Finite Similitude in Fracture Mechanics
}

\author{
Keith Davey, Rooholamin Darvizeh, Jingwen Zhang \\ School of Mechanical, Aerospace and Civil Engineering, \\ The University of Manchester, ManchesterM13 9PL, UK
}

\begin{abstract}
Scaled experimentation can potentially provide significant benefits such as reduced costs materials and time in testing but is afflicted by the phenomena of scale effects, where the behaviour at scale can be markedly different to that at full size. The design of scaled experiments is presently predominantly founded on the theory of dimensional analysis, which itself is grounded on the invariance of dimensionless governing equations with scale. The reality of fracture mechanics however is not invariant equations, but significant deviations and it is evident that scaled-fracture related experiments are presently limited by this affliction.
\end{abstract}

This paper examines an advance in a new approach to scaled experimentation called finite similitude. The key question addressed here is whether it is possible to overcome the affliction of scale effects by performing not one but two scaled experiments at different scales. It is shown that finite similitude (unlike dimensional analysis) is able to capture all forms of scale dependency, which opens up the possibility of selecting alternative forms of scale invariance and consequently alternative forms of similitude.

First-order finite similitude is investigated in the paper and applied to cracked compacttension and three-point bending test specimens along with a cracked pressure vessel to illustrate the new concepts. These case studies reveal the veracity and potential of the new approach and highlight possibilities that hitherto would have been deemed impossible with the circumvention of scale effects (as traditionally defined).

Keywords: fracture mechanics; finite similitude; scaled experimentation. 


\section{Introduction}

Product and component testing is an important practice in many industries and is required to assure in-service reliability and performance. Scaled experimentation has an important role to play in many testing systems but care must be taken to ensure that any results from a scaled experiment are carefully scrutinised. The principal concern with scaling is scale effects where the scaled system behaves in a manner that can be markedly different from the full-size system. It might well be contended that if scale effects were not a feature of scaling, then scaled experimentation would play a far greater role than it presently does. The absence of scale effects would enable complex systems to be more readily analysed at a fraction of the cost of a full-scale trial. Observed phenomena at scale would then replicate that at full size and large-scale reductions would pose no concern; however, the reality is somewhat different.

Despite the presence of scale effects, scaled experiments are performed in industry. There are several reasons for this, but these are largely related to cost and/or the impracticability of full-scale trials. The ever-increasing accuracy and effectiveness of computational modelling has undoubtedly impacted on the type and nature of experiments performed. Scaling is widely applied in different areas of engineering, for example in structural failure and fracture [1-4], structural impact [5-8], explosion engineering [9-10] and thermofluids [11]. A reliable computational model can often replace experiments altogether but in addition it can supplement scaled experimentation. Numerical analysis at scale and at full size can be used to identify and gauge the importance of scale effects [12]. For more complex systems involving significant uncertainties, placing too much reliance on computation is not sensible and experimentation is therefore needed; for instance, the scaling of fluid mechanics [13] or simulation in complex conditions [14].

One of earliest works in structural mechanics on similitude followed some 30 years after Rayleigh's 1915 paper [15] in a US aeronautical advisory committee report by Goodier and Thomson [16]. This was followed by Goodier's book in 1950 [17] covering the applicability of similitude to structural systems. Goodier and Thomson presented the first application of dimensional analysis to relatively simple and more complex structural problems. They examined aspects of large deformation, buckling and plastic behaviour covering aspects of materials with nonlinear stress-strain characteristics. A good review of the many works based on dimensional analysis up to 2000 is provided by Simitses et al. in reference [18].

Despite the wide application of dimensional analysis, it has been shown to suffer some limitations in many studies all stemming from scale effects. Particularly renown for such 
effects is indentation [19], which is of relevance to instrumentation for the measurement of hardness. Even in the early work of Brinell [20] it was observed that the hardness value is sensitive to ball diameter. In this case the difference can be predominantly attributed to size effects arising with the size of the deformation zone and the change in material response because of this. However other scale effects have been identified and these include surface contamination, energy, and roughness along with indenter tip rounding and friction [21].

The behaviour of concrete in fracture is a material that has notoriety for size effects; see for example the work of Bažant [21]. Since scale effects are characterized by the failure of dimensional analysis in the sense that the invariance of the dimensionless governing equations breaks down with scale, it is often used in investigations of scale effects. An example of this is a recent study concerned with scaling and its effects on fatigue and lifetime [22], where dimensional analysis is applied to qualify whether scale effects are present or not. Other studies concerned with fatigue, size effect and the application of dimensional analysis [23-25] again highlight the presence of scale effects through change to the dominant governing dimensionless equation with scale.

This paper is concerned with a new approach to scaled experimentation applied to fracture. The finite-similitude theory [26-28] is founded on the metaphysical concept of space scaling, where it is imagined that space is contracted and all things in the space suffer scaling therefore. Although patently such scaling cannot be achieved practically, what is possible is an examination of the effect that this metaphysical process has on the governing equations constraining the behavior of the phenomena of study. The metaphysics of space scaling is introduced in Section 2 along with its effects on a moving control volume in the space being considered. The immediacy of the effect of space scaling on a control volume, which is simply a region of space, is one of the underlying reasons for describing the equations of interest is an integral transport form. A generic form of transport equation is therefore introduced in Section 3 and the effect space scaling has on it is examined. Critical to the finite similitude approach is the representation of trial-space physics on the full-scale space. It is through this representation that scale dependencies are revealed in either an explicit or implicit form. Moreover, the representation provides a mechanism for the contrasting of behaviors at different scales and in fact defines what is algebraically allowable. This aspect is examined in greater detail in Section 4 under the title of projected continuum mechanics, where trial-space conserved and non-conserved transport equations are examined in their projected form in the full-scale physical space. The proportional relationships typical to dimensional analysis are revealed in this section on application of the simple assumption that 
the projected transport equations do not change with scale. This is equivalent to the condition that the differentiation of the projected equations with respect to the length scalar ( $\beta$ in this paper) is identically zero. The realization that this is just one possibility and other possibilities exist is examined in Section 5. Introduced is high-order finite similitude but focus of this paper is on first order only, where an identity involving two nested derivatives with respect to $\beta$ is involved. Two derivatives signify two scaled experiments but more fundamentally, scale effects as previously defined using dimensional analysis can cease to be scale effects under the new definition. Analytical applications are examined in Section 6 for the $\mathbf{J}$ integral and the cohesive zone model since both concepts play important roles in fracture mechanics. Numerical studies are presented in Sections 7, 8 and 9 to demonstrate the practical benefit of the new scaling approach to fracture mechanics where scale effects (as previously defined) are prevalent. The paper concludes with a list of conclusions.

\section{Background concepts}

Finite similitude is a new scaling theory founded on the metaphysical concept of space scaling. The idea that space can be expanded or contracted for the purpose of scaled experimentation is evidently not feasible but what is possible is the ability to assess the impact of this metaphysical process on the underpinning physics dictating the behaviour of an experiment. In this way it is possible to assess what changes take place as space deforms and design experiments accordingly.

\section{1. $\quad$ Space scaling}

To enact the space-scaling concept consider the affine map $\boldsymbol{x}_{p s} \mapsto \boldsymbol{x}_{t s}$ which relates coordinate points in the inertial frame in the physical space (full size) to those in the trial space (scaled size). Points in each inertial frame are placed in one-to-one correspondence and the map is assumed to be affine and temporally invariant. In differential terms the map provides $\boldsymbol{d} \boldsymbol{x}_{t s}=\boldsymbol{F} \boldsymbol{d} \boldsymbol{x}_{p s}$ where $F$ is both spatially and temporally invariant and in coefficient form is $d x_{t s}^{i}=F^{i}{ }_{j} d x_{p s}^{j}$, where $F^{i}{ }_{j}=\partial x_{t s}^{i} / \partial x_{p s}^{j}$, where $x_{t s}^{i}$ and $x_{p s}^{i}$ are coordinate functions. The matrix $F$ dictates the manner in which space distorts, where anisotropic scaling is possible but focus here is on isotropic scaling, where $F$ takes on the relatively simple form $F=\beta I$ or $F_{j}^{i}=\beta \delta_{j}^{j}$, where $\delta_{j}^{j}$ is the well-known Kronecker delta symbol and takes the value of either zero or one. The scalar $\beta$ is a positive real parameter that quantifies the extent of the scaling involved. For $0<\beta<1$ the space is contracted, for $\beta=1$ no scaling takes 
place and for $\beta>1$ the space is expanded. The space scaling concept is illustrated in in Fig. 1 , where contraction and expansion are depicted.

With the assumed existence of the differential map $\boldsymbol{d} \boldsymbol{x}_{t s}=\beta \boldsymbol{d} \boldsymbol{x}_{p s}$ it is now possible to relate differential volumes and areas in the two spaces. This is achieved readily by means of Nanson's identities, which take the form $d V_{t s}=\beta^{3} d V_{p s}$ and $\boldsymbol{d} \boldsymbol{\Gamma}_{t s}=\beta^{2} \boldsymbol{d} \boldsymbol{\Gamma}_{p s}$, where $\boldsymbol{d} \boldsymbol{\Gamma}_{t s}=$ $\boldsymbol{n}_{t s} d \Gamma_{t s}$, and $\boldsymbol{d} \boldsymbol{\Gamma}_{p s}=\boldsymbol{n}_{p s} d \Gamma_{p s}$, and $\boldsymbol{n}_{t s}$ and $\boldsymbol{n}_{p s}$ are unit normal vectors in the respective spaces. The quantities $d V_{t s}$ and $d V_{p s}$ along with $d \Gamma_{t s}$ and $d \Gamma_{p s}$ are differential measures of volume and area respectively in the two spaces.

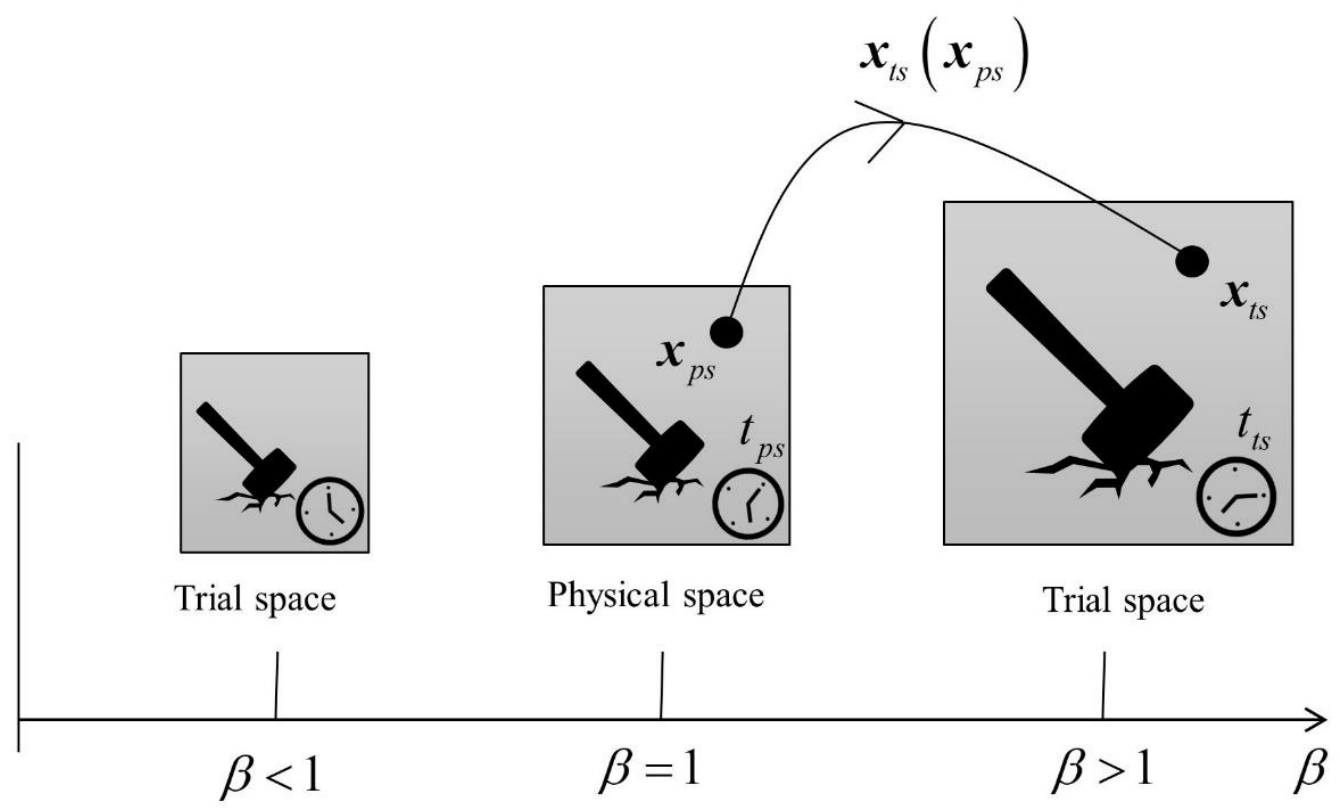

Figure 1 Depiction of space scaling and mapping between inertial physical and trial-space frames.

The adoption of a Newtonian framework means the assumed existence of absolute time, labelled $t_{t s}$ and $t_{p s}$ in the respective spaces. These are assumed to be related by the differential identity $d t_{t s}=g d t_{p s}$, where $g$ is a positive scalar and measures the extent of the difference in rate at which processes proceed in the two spaces.

\subsection{Control volume movement}

With the establishment of space scaling it expedient here to examine what affect this has on the behaviour of the underpinning pertinent physics in a continuum mechanics framework. The physical description adopted here is founded on the control-volume concept as this approach immediately brings into play the effects of the differential measures of volume, area and length arising from space scaling. A control volume is a finite region of space and takes 
the form of a continuous open domain $\Omega_{t s}^{*}$ (in the trial space) whose closure contains the orientable boundary $\Gamma_{t s}^{*}$ with outward pointing unit normal $\boldsymbol{n}_{t s}$. The exact same apparatus applies to the physical space and it should be recognised that both $\Omega_{p s}^{*}$ and $\Omega_{t s}^{*}$ are free to deform and move but in the context of scaled experimentation it can be anticipated that their movement must be synchronised in some manner. The movement of $\Omega_{t s}^{*}$ can be related to the velocity field $\boldsymbol{v}_{t s}^{*}$ using the identity $\boldsymbol{v}_{t s}^{*}=D^{*} \boldsymbol{x}_{t s}^{*} / D^{*} t_{t s}^{*}$, where the temporal derivative $D^{*} / D^{*} t_{t s}^{*}$ is a partial derivative that holds constant points in a reference control volume $\Omega_{t s}^{* r e f}$, i.e. $D^{*} / D^{*} t_{t s}^{*} \equiv \partial /\left.\partial t_{t s}^{*}\right|_{\chi_{t s}}$, where $\chi_{t s} \in \Omega_{t s}^{* r e f}$. In this manner the movement of a coordinate point is precisely described by the solution of the differential equation $\boldsymbol{v}_{t s}^{*}=D^{*} \boldsymbol{x}_{t s}^{*} / D^{*} t_{t s}^{*}$ (with $\boldsymbol{v}_{t s}^{*}$ known) or the map $\Omega_{t s}^{* r e f} \rightarrow \Omega_{t s}^{*}$ (i.e. $\chi_{t s} \mapsto \boldsymbol{x}_{t s}^{*}$ ).

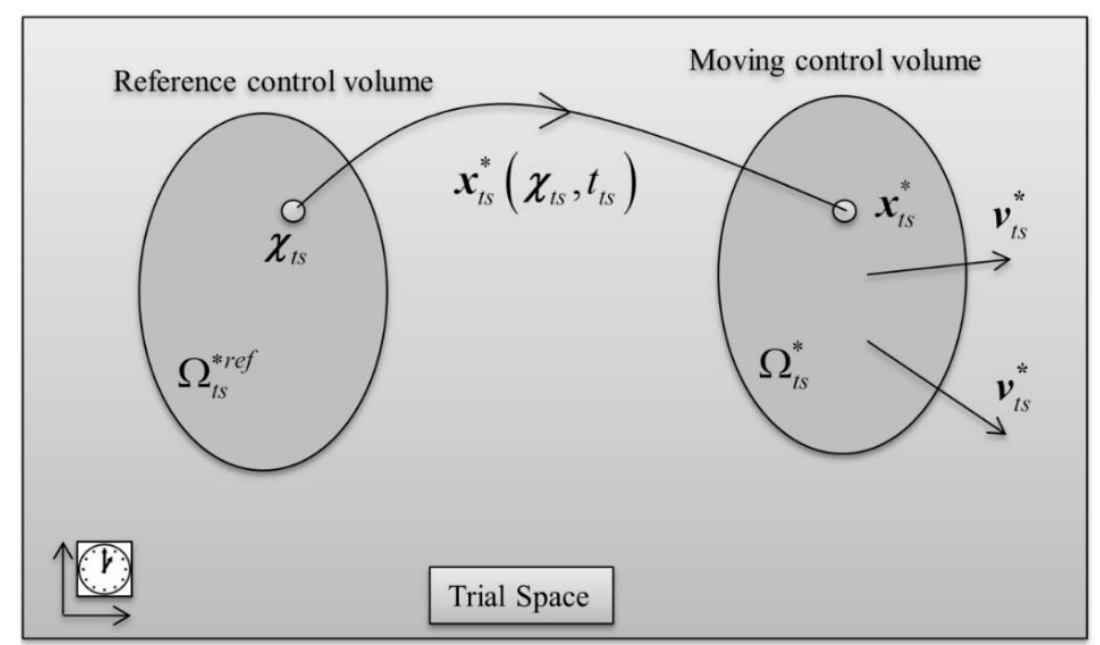

Figure 2 Moving control volume in the trial space and mapping from a reference control volume.

The concept is illustrated in Fig. 2, where a moving control volume of arbitrary shape facilitates the focus on a particular region of space, allowing for changing shape and movement, as necessary. Needless to say the exact same apparatus applies in the physical space with the identity $\boldsymbol{v}_{p s}^{*}=D^{*} \boldsymbol{x}_{p s}^{*} / D^{*} t_{p s}^{*}$. The assumed relationship between $\boldsymbol{x}_{t s}^{*}$ and $\boldsymbol{x}_{p s}^{*}$ suggests a map of the form $\boldsymbol{x}_{p s}^{*} \mapsto \boldsymbol{x}_{t s}^{*}$. This map transpires to be identical in form to the space-scaling map, i.e. $\boldsymbol{d} \boldsymbol{x}_{t s}^{*}=\beta \boldsymbol{d} \boldsymbol{x}_{p s}^{*}$, and in view of the temporal relationship $d t_{t s}=g d t_{p s}$, the velocities $\boldsymbol{v}_{t s}^{*}$ and $\boldsymbol{v}_{p s}^{*}$ are related by $\boldsymbol{v}_{t s}^{*}=\beta g^{-1} \boldsymbol{v}_{p s}^{*}$ and further details on this can be found in reference [26]. The ability to relate control-volume movement without reference to the physical processes in the two spaces is important as it provides for a generic approach.

\section{The projected transport equations}


Due to the relative dominance of variational methods, control-volume approaches involving transport equations in their integral form for applications in solid mechanics are somewhat neglected (see Davey \& Darvizeh [29]). In the field of fracture mechanics in particular the theory of configurational forces [30] does make reference to control-volume ideas although in a material-reference frame and also their use is required in shock physics [31] to capture discontinuous behaviour. The ability to capture all the physical laws relating to continuum physics using the control volume approach is its principal advantage in scaling theory. Moreover, it is made apparent here that all scale dependencies are revealed, and different forms of scale effects arise depending on the how similitude is defined. Transport equations in their most generic form in the physical space can be represented by [29]

$$
\begin{gathered}
\frac{D^{*}}{D^{*} t_{p s}} \int_{\Omega_{p s}^{*}} \rho_{p s} \boldsymbol{\Psi}_{p s} d V_{p s}^{*}+\int_{\Gamma_{p s}^{*}} \rho_{p s} \boldsymbol{\Psi}_{p s}\left(\boldsymbol{v}_{p s}-\boldsymbol{v}_{p s}^{*}\right) \cdot \boldsymbol{n}_{p s} d \Gamma_{p s}^{*} \\
=-\int_{\Gamma_{p s}^{*}} \boldsymbol{J}_{p s}^{\Psi} \cdot \boldsymbol{n}_{p s} d \Gamma_{p s}^{*}+\int_{\Omega_{p s}^{*}} \rho_{p s} \boldsymbol{b}_{p s}^{\Psi} d V_{p s}^{*}
\end{gathered}
$$

where $\rho_{p s}, \boldsymbol{\Psi}_{p s}, \boldsymbol{v}_{p s}, \boldsymbol{J}_{p s}^{\Psi}, \boldsymbol{b}_{p s}^{\Psi}, \boldsymbol{n}_{p s}$ signify material density, physical field, material velocity field, flux, source and unit normal to boundary $\Gamma_{\mathrm{ps}}^{*}$ of the control volume $\Omega_{p s}^{*}$.

Similarly, and somewhat more importantly the trial-space transport equations take the identical form

$$
\begin{gathered}
\frac{D^{*}}{D^{*} t_{t s}} \int_{\Omega_{t s}^{*}} \rho_{t s} \boldsymbol{\Psi}_{t s} d V_{t s}^{*}+\int_{\Gamma_{p s}^{*}} \rho_{t s} \boldsymbol{\Psi}_{p t s}\left(\boldsymbol{v}_{t s}-\boldsymbol{v}_{t s}^{*}\right) \cdot \boldsymbol{n}_{t s} d \Gamma_{t s}^{*} \\
=-\int_{\Gamma_{t s}^{*}} \boldsymbol{J}_{t s}^{\psi} \cdot \boldsymbol{n}_{t s} d \Gamma_{t s}^{*}+\int_{\Omega_{t s}^{*}} \rho_{t s} \boldsymbol{b}_{t s}^{\psi} d V_{t s}^{*}
\end{gathered}
$$

where on substitution of the identities $d V_{t s}^{*}=\beta^{3} d V_{p s}^{*}, \boldsymbol{d} \boldsymbol{\Gamma}_{t s}^{*}=\beta^{2} \boldsymbol{d} \boldsymbol{\Gamma}_{p s}^{*}, d t_{t s}=g d t_{p s}$, and on multiplication throughout by g and a scalar $\alpha_{0}^{\Psi}$, gives rise to the critically important equation

$$
\begin{aligned}
\alpha_{0}^{\Psi} T_{0}^{\Psi}(\beta)= & \frac{D^{*}}{D^{*}} t_{p s} \int_{\Omega_{p s}^{*}} \alpha_{0}^{\Psi} \rho_{t s} \beta^{3} \boldsymbol{\Psi}_{t s} d V_{p s}^{*} \\
& +\int_{\Gamma_{p s}^{*}} \alpha_{0}^{\Psi} \rho_{t s} \beta^{3} \boldsymbol{\Psi}_{t s}\left(\beta^{-1} g \boldsymbol{v}_{t s}-\beta^{-1} g \boldsymbol{v}_{t s}^{*}\right) \cdot \boldsymbol{n}_{p s} d \Gamma_{p s}^{*} \\
& +\int_{\Gamma_{p s}^{*}} \alpha_{0}^{\Psi} \beta^{2} g \boldsymbol{J}_{t s}^{\Psi} \cdot \boldsymbol{n}_{p s} d \Gamma_{p s}^{*}-\int_{\Omega_{t s}^{*}} \alpha_{0}^{\Psi} \rho_{t s} \beta^{3} g \boldsymbol{b}_{t s}^{\Psi} d V_{p s}^{*}=0
\end{aligned}
$$


which is essentially Eq. (2) but represented now on the physical space, where the scalar $\alpha_{0}^{\Psi}$ along with $g$ are assumed to be functions of $\beta$.

This equation is fundamental to the finite-similitude theory as it captures in one form or another all scale dependencies. The fields, fluxes and sources are assumed dependent on $\beta$ and have the property that $\rho_{t s}(1)=\rho_{p s}, \boldsymbol{v}_{t s}(1)=\boldsymbol{v}_{p s}, \boldsymbol{\Psi}_{t s}(1)=\boldsymbol{\Psi}_{p s}, \boldsymbol{J}_{t s}(1)=\boldsymbol{J}_{p s}$ and $\boldsymbol{b}_{t s}(1)=\boldsymbol{b}_{p s}$, and similarly in order for Eq. (3) to match Eq. (1) for $\beta=1$ it is required that

$\alpha_{0}^{\Psi}(1)=1$ and $g(1)=1$. It should be appreciated the equation $\alpha_{0}^{\Psi} T_{0}^{\Psi}(\beta)=0$ is not an approximation as it is representative of the trial-space physics but projected onto the physical space and it is through this projection that all $\beta$-dependencies are revealed. Some of these dependencies are explicit such as those arising from geometrical changes (i.e. $\beta^{3}$ and $\beta^{2}$ terms) yet others are implicit (i.e. $\rho_{t s}(\beta), \boldsymbol{v}_{t s}(\beta), \boldsymbol{\Psi}_{t s}(\beta), \boldsymbol{J}_{t s}(\beta)$ and $\boldsymbol{b}_{t s}(\beta)$ ).

\section{Projected continuum mechanics}

Fracture mechanics predominantly makes use of Newtonian mechanics (i.e. three equations) but in scaling theory eight transport equations are to be considered, i.e. two scalar equations for volume and mass conservation, and two vector equations momentum and movement. Although movement is a feature of fracture mechanics it is seldom considered in transport form and was first introduced by Davey and Darvizeh [29] in order to bring the displacement field $\boldsymbol{u}_{p s}$ into the family of transport equations for continuum mechanics. In summary the eight equations are:

$$
\begin{gathered}
\alpha_{0}^{1} T_{0}^{1}(\beta)=\frac{D^{*}}{D^{*} t_{p s}} \int_{\Omega_{p s}^{*}} \alpha_{0}^{1} \beta^{3} d V_{p s}^{*}-\int_{\Gamma_{p s}^{*}} \alpha_{0}^{1} \beta^{3}\left(\beta^{-1} g \boldsymbol{v}_{t s}^{*} \cdot \boldsymbol{n}_{p s}\right) d \Gamma_{p s}^{*}=0 \\
\alpha_{0}^{\rho} T_{0}^{\rho}(\beta)=\frac{D^{*}}{D^{*} t_{p s}} \int_{\Omega_{p s}^{*}} \alpha_{0}^{\rho} \rho_{t s} \beta^{3} d V_{p s}^{*} \\
\quad+\int_{\Gamma_{p s}^{*}} \alpha_{0}^{\rho} \rho_{t s} \beta^{3}\left(\beta^{-1} g \boldsymbol{v}_{t s}-\beta^{-1} g \boldsymbol{v}_{t s}^{*}\right) \cdot \boldsymbol{n}_{p s} d \Gamma_{p s}^{*}=0
\end{gathered}
$$




$$
\begin{aligned}
& \alpha_{0}^{v} T_{0}^{v}(\beta)=\frac{D^{*}}{D^{*} t_{p s}} \int_{\Omega_{p s}^{*}}\left[\alpha_{0}^{v} g^{-1} \beta \rho_{t s} \beta^{3}\right]\left(\beta^{-1} g \boldsymbol{v}_{t s}\right) d V_{p s}^{*} \\
& \quad+\int_{\Gamma_{p s}^{*}}\left[\alpha_{0}^{v} g^{-1} \beta \rho_{t s} \beta^{3}\right]\left(\beta^{-1} g \boldsymbol{v}_{t s}\right)\left(\beta^{-1} g \boldsymbol{v}_{t s}-\beta^{-1} g \boldsymbol{v}_{t s}^{*}\right) \cdot \boldsymbol{n}_{p s} d \Gamma_{p s}^{*} \\
& \quad+\int_{\Gamma_{p s}^{*}} \alpha_{0}^{v} \beta^{2} g \boldsymbol{\sigma}_{t s} \cdot \boldsymbol{n}_{p s} d \Gamma_{p s}^{*}-\int_{\Omega_{t s}^{*}} \alpha_{0}^{v} \rho_{t s} \beta^{3} g \boldsymbol{b}_{t s}^{v} d V_{p s}^{*}=0 \\
& \alpha_{0}^{u} T_{0}^{u}(\beta)=\frac{D^{*}}{D^{*} t_{p s}} \int_{\Omega_{p s}^{*}} \alpha_{0}^{u} \beta \rho_{t s} \beta^{3}\left(\beta^{-1} \boldsymbol{u}_{t s}\right) d V_{p s}^{*}+\int_{\Gamma_{p s}^{*}} \alpha_{0}^{u} \beta \rho_{t s} \beta^{3}\left(\beta^{-1} \boldsymbol{u}_{t s}\right)\left(\beta^{-1} g \boldsymbol{v}_{t s}-\right. \\
& \left.\beta^{-1} g \boldsymbol{v}_{t s}^{*}\right) \cdot \boldsymbol{n}_{p s} d \Gamma_{p s}^{*}-\int_{\Omega_{t s}^{*}} \alpha_{0}^{u} \beta \rho_{t s} \beta^{3}\left(\beta^{-1} g \boldsymbol{v}_{t s}\right) d V_{p s}^{*}=0
\end{aligned}
$$

where to satisfy the zeroth-order identity

\begin{tabular}{|c|c|c|c|c|c|}
\hline $\begin{array}{l}\text { Eq. } \\
\text { No. }\end{array}$ & Field & Scalars & Flux & Source & Duplicate \\
\hline$(4 a)$ & $\boldsymbol{v}_{t s}^{*}=\beta g^{-1} \boldsymbol{v}_{p s}^{*}$ & $\alpha_{0}^{1}=\beta^{-3}$ & - & - & - \\
\hline$(4 b)$ & $\alpha_{0}^{\rho} \rho_{t s} \beta^{3}=\rho_{p s}$ & $\begin{array}{c}\alpha_{0}^{\rho}(1)=1 \\
g(1)=1\end{array}$ & & & $\begin{array}{l}\boldsymbol{v}_{t s}=\beta g^{-1} \boldsymbol{v}_{p s} \\
\boldsymbol{v}_{t s}^{*}=\beta g^{-1} \boldsymbol{v}_{p s}^{*}\end{array}$ \\
\hline$(4 c)$ & $\boldsymbol{v}_{t s}=\beta g^{-1} \boldsymbol{v}_{p s}$ & $\alpha_{0}^{v}=\alpha_{0}^{\rho} g \beta^{-1}$ & $\begin{array}{l}\alpha_{0}^{v} \beta^{2} g \boldsymbol{\sigma}_{t s} \\
=\boldsymbol{\sigma}_{p s}\end{array}$ & $\begin{array}{l}\alpha_{0}^{v} \rho_{t s} \beta^{3} g \boldsymbol{b}_{t s}^{v} \\
=\rho_{t s} \boldsymbol{b}_{p s}^{v}\end{array}$ & $\boldsymbol{v}_{t s}^{*}=\beta g^{-1} \boldsymbol{v}_{p s}^{*}$ \\
\hline$(4 d)$ & $\boldsymbol{u}_{t s}=\beta \boldsymbol{u}_{p s}$ & $\alpha_{0}^{u}=\alpha_{0}^{\rho} \beta^{-1}$ & & $\begin{array}{l}\boldsymbol{v}_{t s} \\
=\beta g^{-1} \boldsymbol{v}_{p s}\end{array}$ & $\boldsymbol{v}_{t s}^{*}=\beta g^{-1} \boldsymbol{v}_{p s}^{*}$ \\
\hline
\end{tabular}

$$
\frac{d}{d \beta}\left(\alpha_{0}^{\Psi} T_{0}^{\Psi}\right) \equiv 0
$$

the necessary and sufficient identities arising from these equations are presented in Table 1.

Table 1: Necessary and sufficient zeroth-order scaling identities.

Table 1 presents those identities arising from Eqs. (4) because of Eq. (5), which is the situation where scale effects are absent. An additional "Duplicate" column is included in Table 1 to capture those field identities that appear in more than one equation. Observe that 
the role of Eq. (4a) is the determination of the identity $\boldsymbol{v}_{t s}^{*}=\beta g^{-1} \boldsymbol{v}_{p s}^{*}$, which is required so that control-volume movement in the trial space at any scale can be described in the physical space. In this sense the behaviour of the trial-space system is played out on the physical space enabling differences to be gauged. In order to satisfy Eq. (5) for Eq. (4b) it is necessary and sufficient to set $\alpha_{0}^{\rho} \rho_{t s} \beta^{3}=\rho_{p s}$ and $\boldsymbol{v}_{t s}=\beta g^{-1} \boldsymbol{v}_{p s}$ (along with $\boldsymbol{v}_{t s}^{*}=$ $\left.\beta g^{-1} \boldsymbol{v}_{p s}^{*}\right)$ with $\alpha_{0}^{\rho}(1)=1$ and $\boldsymbol{v}_{t s}(1)=\boldsymbol{v}_{p s}$. Although continuity plays little part in fracture mechanics it is needed for similitude to establish a relationship for density with scale (i.e. $\alpha_{0}^{\rho} \rho_{t s} \beta^{3}=\rho_{p s}$ ), which opens up the possibility of selecting alternative materials for a scaled experiment. The velocity relationship $\boldsymbol{v}_{t s}=\beta g^{-1} \boldsymbol{v}_{p s}$ is particularly constraining and it is effectively restricting all scaled experiments to the same pattern of deformation, which is unrealistic in practice. The momentum transport Eq. (4c) has a critical role to place in fracture mechanics and to satisfy Eq. (5) with $\alpha_{0}^{\rho} \rho_{t s} \beta^{3}=\rho_{p s}$ it is necessary and sufficient to set $\alpha_{0}^{v}=\alpha_{0}^{\rho} g \beta^{-1}$ (as above $\boldsymbol{v}_{t s}=\beta g^{-1} \boldsymbol{v}_{p s}$ and $\boldsymbol{v}_{t s}^{*}=\beta g^{-1} \boldsymbol{v}_{p s}^{*}$ ), $\alpha_{0}^{v} \beta^{2} g \boldsymbol{\sigma}_{t s}=\boldsymbol{\sigma}_{p s}$ and $\alpha_{0}^{v} \rho_{t s} \beta^{3} g \boldsymbol{b}_{t s}^{v}=\rho_{t s} \boldsymbol{b}_{p s}^{v}$; note that $\alpha_{0}^{v}(1)=1$. Again in view of the relationship $\alpha_{0}^{\rho} \rho_{t s} \beta^{3}=$ $\rho_{p s}$ it is necessary and sufficient in the movement equation, Eq. (4d) to set $\alpha_{0}^{u}=\alpha_{0}^{\rho} \beta^{-1}$ (as above $\boldsymbol{v}_{t s}=\beta g^{-1} \boldsymbol{v}_{p s}$ and $\left.\boldsymbol{v}_{t s}^{*}=\beta g^{-1} \boldsymbol{v}_{p s}^{*}\right)$ and $\boldsymbol{u}_{t s}=\beta \boldsymbol{u}_{p s}$; note also that $\alpha_{0}^{u}(1)=1$ and $\boldsymbol{u}_{t s}(1)=\boldsymbol{u}_{p s}$. The relationship for displacement is not too unexpected since differentiation of $\boldsymbol{u}_{t s}=\beta \boldsymbol{u}_{p s}$ with respect to time (given the identity $d t_{t s}=g d t_{p s}$ ) provides as required $\boldsymbol{v}_{t s}=\beta g^{-1} \boldsymbol{v}_{p s}$. Note also that the two identities $\boldsymbol{u}_{t s}=\beta \boldsymbol{u}_{p s}$ and $\boldsymbol{d} \boldsymbol{x}_{t s}=\beta \boldsymbol{d} \boldsymbol{x}_{p s}$ provide the small-strain tensor identity $\boldsymbol{\varepsilon}_{t s}=\boldsymbol{\varepsilon}_{p s}$, which immediately infers that the strain tensor $\boldsymbol{\varepsilon}_{t s}$ is independent of $\beta$.

Overall, it is fairly evident that identity Eq. (5) is very restrictive on the behaviour of the trialspace systems and unlikely to be satisfied for realistic problems. See previous studies on the practical application of zeroth-order finite similitude theory in references [32-34]. The reality in practice therefore is the inequality

$$
\frac{d}{d \beta}\left(\alpha_{0}^{\Psi} T_{0}^{\psi}\right) \not \equiv 0
$$

which of course provides scale effects, and these can be expected to change with scale, i.e. be dependent on $\beta$.

\section{First-order finite similitude}


Eq. (3) provides the framework for the analysis of scale dependence but contains hidden dependencies that require information on such things such as material behaviour (e.g. size dependence) and boundary conditions (e.g. surface conditions) to uncover them. However, an alternative (and the approach adopted here) is to simply enforce a global $\beta$ - invariant condition and apply this in the design of experiments. First-order finite similitude [35] examines an alternative to Eq. (5) that involves an additional scaled experiment to shed additional light on changes that are taking place and to add extra flexibility. Consider then the following definition:

\subsection{Definition (High-order finite similitude)}

The concept of $\mathrm{k}^{\text {th }}$-order finite similitude is identified by the lowest derivative that satisfies

$$
T_{k+1}^{\Psi}=\frac{d}{d \beta}\left(\alpha_{k}^{\Psi} T_{k}^{\Psi}\right) \equiv 0
$$

$\forall \beta>0$, with $\alpha_{0}^{\Psi} T_{0}^{\Psi}$ defined by Eq. (3) and non-zero scalars $\alpha_{k}^{\Psi}$ are functions of $\beta$ with $\alpha_{k}^{\Psi}(1)=1$, where the sign " $\equiv$ " means identically zero in Eq. (7).

The corresponding scaling theory is termed $\mathrm{k}^{\text {th }}$-order finite similitude but the focus in this paper is on first-order finite similitude only, i.e.

$$
T_{2}^{\Psi}=\frac{d}{d \beta}\left(\alpha_{1}^{\Psi} T_{1}^{\Psi}\right)=\frac{d}{d \beta}\left(\alpha_{1}^{\Psi} \frac{d}{d \beta}\left(\alpha_{0}^{\Psi} T_{0}^{\Psi}\right)\right) \equiv 0
$$

where $\alpha_{1}^{\Psi}$ is required to play a role similar to that of $\alpha_{0}^{\Psi}$ in the annihilation of $\beta$ terms to facilitate the satisfaction of this identity.

Under the new definition zeroth-order finite similitude as discussed in Section 4 is identified by the identity $T_{1}^{\Psi} \equiv 0$. Recognising the reality of practical testing is scale effects and the inequality $T_{1}^{\Psi} \not \equiv 0$ and recognising the dependence $T_{1}^{\Psi}(\beta)$ motivates the need for something more suitable. Definition 5.1 is designed to provide a pragmatic way forward in a situation where specific information on scale effects is absent. Since it is necessary to integrate the similitude identity to link experiments the definition involving nested derivatives is particularly convenient. A desirable feature of the definition is the nesting of similitude orders, so if zeroth-order finite similitude $\left(T_{1}^{\Psi} \equiv 0\right)$ applies, then first order $\left(T_{2}^{\Psi} \equiv 0\right)$ immediately follows from Eq. (8). Yet another feature is apparent on examination of Eq. (8) arising from its foundation on transport equations in the trial space in the form $\alpha_{0}^{\Psi} T_{0}^{\Psi}=0$, which immediately infers that $\alpha_{1}^{\Psi} T_{1}^{\Psi}=0$ and consequently a different set of transport equations arise. The fields associated with this new set of transport equations are the 
derivatives of the fields in $\alpha_{0}^{\Psi} T_{0}^{\Psi}=0$ with respect to $\beta$. This aspect is not pursued here as integration of Eq. (8) is ultimately required to relate experiments at different scales. The overall idea for three scales $\beta_{2}, \beta_{1}$ and $\beta_{0}=1$ is depicted in Fig. 3., where trial space behaviour is played out on the physical space at each scale. In the following section it is shown how projected equations are combined to provide a virtual replica of the full-scale process.

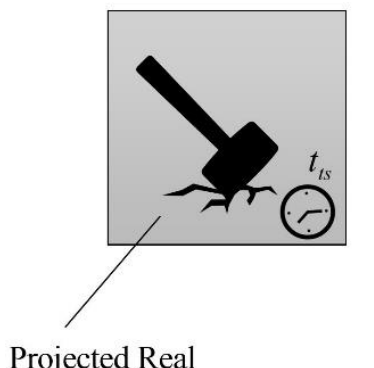

Projected Real

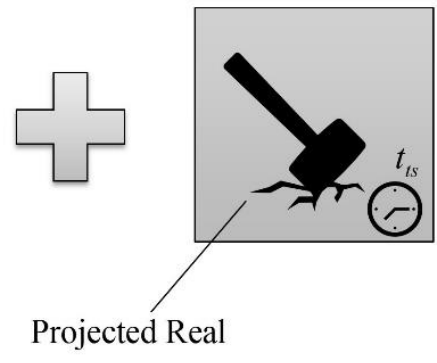

ted Real

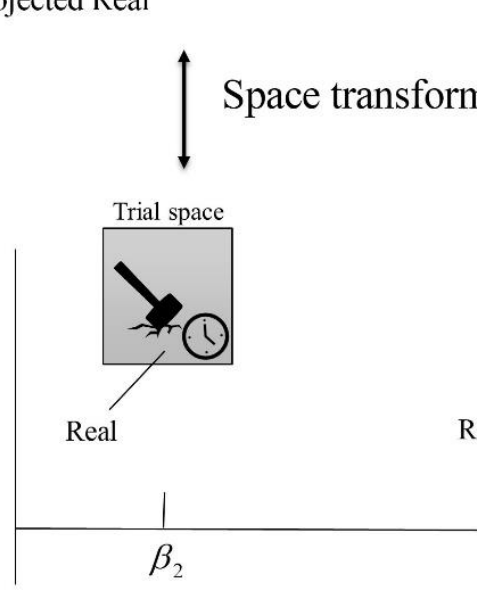

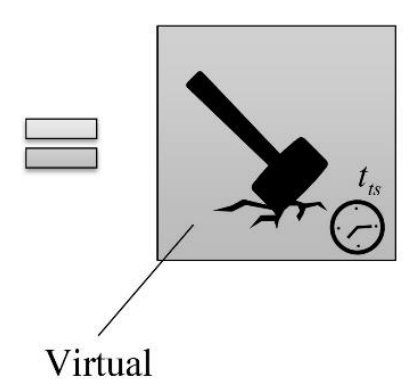

Virtual

(1)

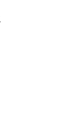
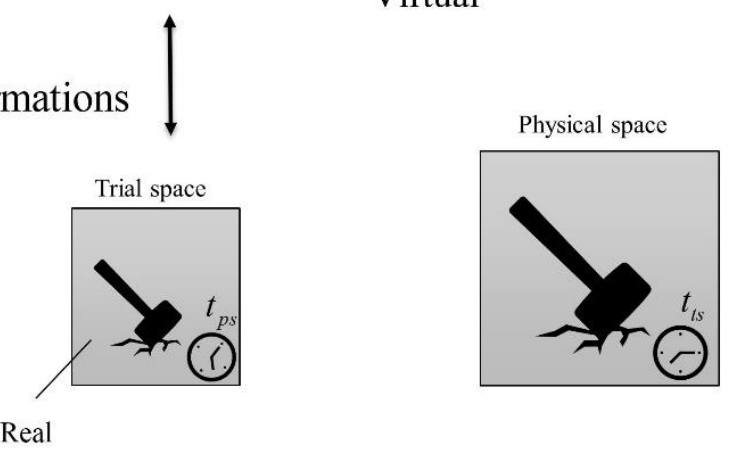

Figure 3. Projected trial-space behaviour described on a control volume at scale $\beta_{i}$ by transport equations $\alpha_{0}^{\Psi} T_{0}^{\Psi}\left(\beta_{i}\right)=0$ and combined to provide a full-scale virtual model.

\subsection{Integrated similitude conditions}

The reality of fracture-mechanics experimentation is discrete experiments at different scales, which invariably means that derivatives with respect to $\beta$ are required to be evaluated by means of finite differences. There exist many forms finite differences can take but for down scaling with $0<\beta \leq 1$ and considering that predicting the behaviour of systems at $\beta=1$ is of prime importance, backward difference formulations naturally emerge. First-order finite similitude involves three scales $\beta_{2}, \beta_{1}$ and $\beta_{0}=1$, and the identity $T_{2}^{\Psi}=d\left(\alpha_{1}^{\Psi} T_{1}^{\Psi}\right) / d \beta \equiv 0$. In order to evaluate this expression consider then the divided-difference table for $\alpha_{0}^{\Psi} T_{0}^{\Psi}$ with three data points $\left\{\beta_{2}, \beta_{1}, \beta_{0}\right\}$, where the first-divided difference and important mean-value identities (mean-value theorem for derivatives) are: 


$$
\begin{aligned}
& \left.\alpha_{1}^{\Psi}\right|_{\widehat{\beta}_{2}^{1}} \frac{\alpha_{0}^{\Psi} T_{0}^{\Psi}\left(\beta_{1}\right)-\alpha_{0}^{\Psi} T_{0}^{\Psi}\left(\beta_{2}\right)}{\beta_{1}-\beta_{2}}=\left.\alpha_{1}^{\Psi}\right|_{\widehat{\beta}_{2}^{1}} T_{1}^{\Psi}\left(\hat{\beta}_{2}^{1}\right) \\
& \left.\alpha_{1}^{\Psi}\right|_{\widehat{\beta}_{1}^{o}} \frac{\alpha_{0}^{\Psi} T_{0}^{\Psi}\left(\beta_{0}\right)-\alpha_{0}^{\Psi} T_{0}^{\Psi}\left(\beta_{1}\right)}{\beta_{0}-\beta_{1}}=\left.\alpha_{1}^{\Psi}\right|_{\widehat{\beta}_{1}^{o}} T_{1}^{\Psi}\left(\hat{\beta}_{1}^{o}\right)
\end{aligned}
$$

where $\beta_{i} \leq \hat{\beta}_{i}^{i-1} \leq \beta_{i-1}$ and bearing in mind that the next divided difference is

$$
\frac{\left.\alpha_{1}^{\Psi}\right|_{\widehat{\beta}_{1}^{o}} T_{1}^{\Psi}\left(\hat{\beta}_{1}^{o}\right)-\left.\alpha_{1}^{\Psi}\right|_{\widehat{\beta}_{2}^{1}} T_{1}^{\Psi}\left(\hat{\beta}_{2}^{1}\right)}{\hat{\beta}_{1}^{o}-\hat{\beta}_{2}^{1}} \equiv 0
$$

which for first-order finite similitude is identically zero and on substitution of Eqs. (9) provides

$$
\alpha_{0}^{\Psi} T_{0}^{\Psi}\left(\beta_{0}\right) \equiv \alpha_{0}^{\Psi} T_{0}^{\Psi}\left(\beta_{1}\right)+R_{1}^{\Psi}\left(\alpha_{0}^{\Psi} T_{0}^{\Psi}\left(\beta_{1}\right)-\alpha_{0}^{\Psi} T_{0}^{\Psi}\left(\beta_{2}\right)\right)
$$

where the scaling parameter $R_{1}^{\Psi}$ in this equation is

$$
R_{1}^{\Psi}=\left(\frac{\left.\alpha_{1}^{\Psi}\right|_{\widehat{\beta}_{2}^{1}}}{\left.\alpha_{1}^{\Psi}\right|_{\widehat{\beta}_{1}^{o}}}\right)\left(\frac{\beta_{0}-\beta_{1}}{\beta_{1}-\beta_{2}}\right)
$$

Examination of Eq. (11) reveals several important aspects. First, first-order finite similitude is about proportional relationships between the differences in the transport equations at different scales. Second, since $\alpha_{0}^{\Psi} T_{0}^{\Psi}\left(\beta_{1}\right)=0$ and $\alpha_{0}^{\Psi} T_{0}^{\Psi}\left(\beta_{2}\right)=0$ it immediately follows from Eq. (11) that $\alpha_{0}^{\Psi} T_{0}^{\Psi}\left(\beta_{0}\right)=0$, i.e. a set of transport equations for the physical space is obtained. Note additionally if zeroth-order similitude applies, then Eq. (11) reduces to

$$
\alpha_{0}^{\Psi} T_{0}^{\Psi}\left(\beta_{0}\right) \equiv \alpha_{0}^{\Psi} T_{0}^{\Psi}\left(\beta_{1}\right)
$$

which is the expected form being the integrated form of Eq. (5).

\subsection{First-order identities}

Eq. (11) gives rise to a set of first order identities on application to Eqs. (4a) to (4c), which are:

$$
\begin{gathered}
\boldsymbol{v}_{p s}=\beta_{1}^{-1} g_{1} \boldsymbol{v}_{t s 1}+R_{1}^{\rho}\left(\beta_{1}^{-1} g_{1} \boldsymbol{v}_{t s 1}-\beta_{2}^{-1} g_{2} \boldsymbol{v}_{t s 2}\right) \\
\boldsymbol{v}_{p s}=\beta_{1}^{-1} g_{1} \boldsymbol{v}_{t s 1}+R_{1}^{v}\left(\beta_{1}^{-1} g_{1} \boldsymbol{v}_{t s 1}-\beta_{2}^{-1} g_{2} \boldsymbol{v}_{t s 2}\right) \\
\boldsymbol{\sigma}_{p s}=\alpha_{01}^{v} g_{1} \beta_{1}^{2} \boldsymbol{\sigma}_{t s 1}+R_{1}^{v}\left(\alpha_{01}^{v} g_{1} \beta_{1}^{2} \boldsymbol{\sigma}_{t s 1}-\alpha_{02}^{v} g_{2} \beta_{2}^{2} \boldsymbol{\sigma}_{t s 2}\right) \\
\boldsymbol{b}_{p s}^{v}=g_{1}^{2} \beta_{1}^{-1} \boldsymbol{b}_{t s 1}^{v}+R_{1}^{v}\left(g_{1}^{2} \beta_{1}^{-1} \boldsymbol{b}_{t s 1}^{v}-g_{2}^{2} \beta_{2}^{-1} \boldsymbol{b}_{t s 2}^{v}\right) \\
\boldsymbol{u}_{p s}=\beta_{1}^{-1} \boldsymbol{u}_{t s 1}+R_{1}^{u}\left(\beta_{1}^{-1} \boldsymbol{u}_{t s 1}-\beta_{2}^{-1} \boldsymbol{u}_{t s 2}\right) \\
\boldsymbol{v}_{p s}=\beta_{1}^{-1} g_{1} \boldsymbol{v}_{t s 1}+R_{1}^{u}\left(\beta_{1}^{-1} g_{1} \boldsymbol{v}_{t s 1}-\beta_{2}^{-1} g_{2} \boldsymbol{v}_{t s 2}\right)
\end{gathered}
$$


and where $\boldsymbol{\sigma}_{t s 1}=\boldsymbol{\sigma}_{t s}\left(\beta_{1}\right), \boldsymbol{v}_{t s 2}=\boldsymbol{v}_{t s}\left(\beta_{2}\right)$ etc. and a consistent velocity field requires $R_{1}^{u}=$ $R_{1}^{v}=R_{1}^{\rho}$ and set to be $R_{1}$ henceforth.

Note that Eq. (14e) along with the identities $\boldsymbol{d} x_{t s 1}=\beta_{1} \boldsymbol{d} x_{p s}$ and $\boldsymbol{d} x_{t s 2}=\beta_{2} \boldsymbol{d} x_{p s}$ yield the small strain relationship

$$
\varepsilon_{p s}=\varepsilon_{t s 1}+R_{1}^{u}\left(\varepsilon_{t s 1}-\varepsilon_{t s 2}\right)
$$

which confirms that to a limited degree, strain is permitted to be unequal in the trial and physical spaces, which is the reality in most physical experiments.

The identities pertaining to first-order finite similitude theory are summarised in Table 2 and revealed is that despite the initial complexity of the theory, relatively straightforward relationships are its product.

Table 2: Necessary and sufficient first-order scaling identities.

\begin{tabular}{|c|c|c|c|c|}
\hline $\begin{array}{l}\text { Eq. } \\
\text { No. }\end{array}$ & Field & Scalars & Flux & Source \\
\hline (4a) & $\boldsymbol{v}_{t s}^{*}=\beta g^{-1} \boldsymbol{v}_{p s}^{*}$ & $\alpha_{0}^{1}=\beta^{-3}$ & - & - \\
\hline (4b) & $\alpha_{0}^{\rho} \rho_{t s} \beta^{3}=\rho_{p s}$ & $\begin{array}{c}\alpha_{0}^{\rho}(1)=1 \\
g(1)=1\end{array}$ & & \\
\hline$(4 c)$ & $\begin{array}{l}\boldsymbol{v}_{p s} \\
=\beta_{1}^{-1} g_{1} \boldsymbol{v}_{t s 1} \\
+R_{1}\left(\beta_{1}^{-1} g_{1} \boldsymbol{v}_{t s 1}\right. \\
\left.-\beta_{2}^{-1} g_{2} \boldsymbol{v}_{t s 2}\right)\end{array}$ & $\alpha_{0}^{v}=\alpha_{0}^{\rho} g \beta^{-1}$ & $\begin{array}{l}\boldsymbol{\sigma}_{p s} \\
=\alpha_{01}^{v} g_{1} \beta_{1}^{2} \boldsymbol{\sigma}_{t s 1} \\
+R_{1}\left(\alpha_{01}^{v} g_{1} \beta_{1}^{2} \boldsymbol{\sigma}_{t s 1}\right. \\
\left.-\alpha_{02}^{v} g_{2} \beta_{2}^{2} \boldsymbol{\sigma}_{t s 2}\right)\end{array}$ & $\begin{array}{l}\boldsymbol{b}_{p s}^{v} \\
=g_{1}^{2} \beta_{1}^{-1} \boldsymbol{b}_{t s 1}^{v} \\
+R_{1}\left(g_{1}^{2} \beta_{1}^{-1} \boldsymbol{b}_{t s}^{v}\right. \\
\left.-g_{2}^{2} \beta_{2}^{-1} \boldsymbol{b}_{t s 2}^{v}\right)\end{array}$ \\
\hline$(4 d)$ & $\begin{array}{l}\boldsymbol{u}_{p s} \\
=\beta_{1}^{-1} \boldsymbol{u}_{t s 1} \\
+R_{1}\left(\beta_{1}^{-1} \boldsymbol{u}_{t s 1}\right. \\
\left.-\beta_{2}^{-1} \boldsymbol{u}_{t s 2}\right)\end{array}$ & $\alpha_{0}^{u}=\alpha_{0}^{\rho} \beta^{-1}$ & & $\begin{array}{l}\boldsymbol{v}_{p s} \\
=\beta_{1}^{-1} g_{1} \boldsymbol{v}_{t s 1} \\
+R_{1}\left(\beta_{1}^{-1} g_{1} \boldsymbol{v}_{t s 1}\right. \\
\left.-\beta_{2}^{-1} g_{2} \boldsymbol{v}_{t s 2}\right)\end{array}$ \\
\hline
\end{tabular}

\section{Analytical fracture studies}


This section is concerned with the application of the new scaling concepts to two important concepts in fracture mechanics, i.e. the J-integral and the cohesive zone model. Depicted in Fig. 4 is the compact tension (CT) test, which is one of the most commonly used specimens in J-integral facture mechanics and forms the main focus in this section.

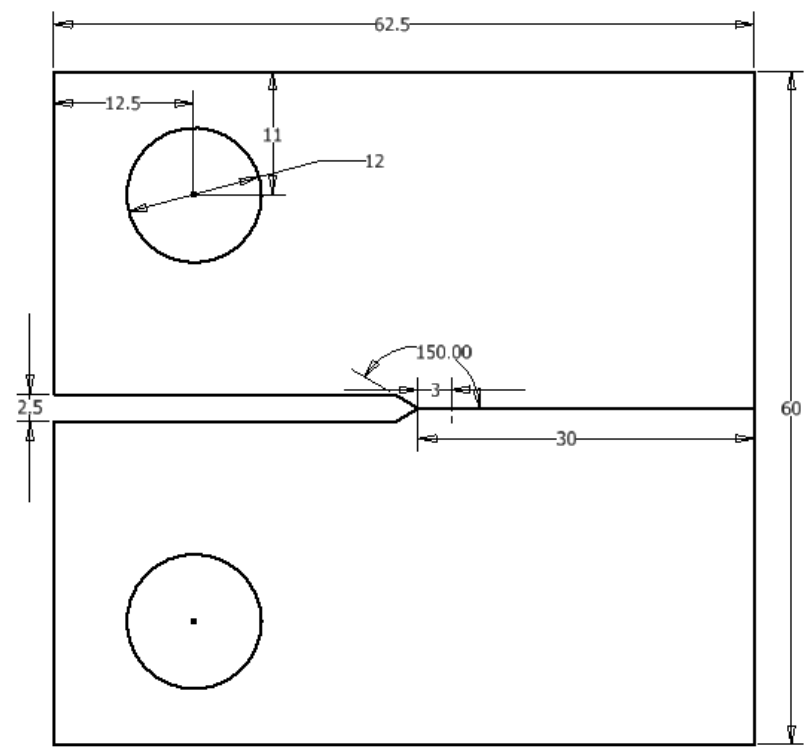

Figure 4. Typical testing arrangement for a compact tesion (CT) specimen.

\subsection{The J-Integral}

The J-integral on a moving control volume traversing a loaded body in equilibrium (in a trial space) with velocity $\boldsymbol{v}_{t s}^{*}$ is provided by Davey and Darvizeh in reference [29] in an extended form

$$
J_{t s}^{*}=\int_{\Gamma_{t s}^{*}} \omega_{t s} \boldsymbol{v}_{t s}^{*} \cdot \boldsymbol{n}_{t s} d \Gamma_{p s}^{*}-\int_{\Gamma_{t s}^{*}}\left(\boldsymbol{v}_{t s}^{*} \cdot \nabla_{t s} \boldsymbol{u}_{t s}\right) \cdot \boldsymbol{\tau}_{t s} d \Gamma_{t s}^{*}
$$

where $\omega_{t s}$ is strain-energy density and $\boldsymbol{\tau}_{t s}$ is traction (i.e. $\boldsymbol{\tau}_{t s}=\boldsymbol{\sigma}_{t s} \cdot \boldsymbol{n}_{t s}$ ), which yields the standard J-integral form [36] on setting $\boldsymbol{v}_{t s}^{*}=v_{t s}^{*} \boldsymbol{e}_{i}$ with $\boldsymbol{e}_{i}$ indicating the direction of crack travel and $v_{t s}^{*}$ is a uniformly invariant speed.

Substitution of the identities $d V_{t s}^{*}=\beta^{2} d V_{p s}^{*}$ and $\boldsymbol{d} \boldsymbol{\Gamma}_{t s}^{*}=\beta \boldsymbol{d} \Gamma_{p s}^{*}$ (note 2-D here) and on multiplication throughout by $\mathrm{g}, \beta^{-1}$ and a scalar $\alpha_{0}^{\omega}$, gives rise to 


$$
\begin{aligned}
\alpha_{0}^{\omega} g \beta^{-1} J_{t s}^{*}= & \int_{\Gamma_{p s}^{*}} \alpha_{0}^{\omega} \omega_{t s} \beta\left(g \beta^{-1} \boldsymbol{v}_{t s}^{*}\right) \cdot \boldsymbol{n}_{p s} d \Gamma_{p s}^{*} \\
& -\int_{\Gamma_{p s}^{*}} \alpha_{0}^{\omega}\left(\alpha_{0}^{v}\right)^{-1} g^{-1} \beta^{-1}\left(\left(g \beta^{-1} \boldsymbol{v}_{t s}^{*}\right) \cdot \nabla_{p s} \beta^{-1} \boldsymbol{u}_{t s}\right) \\
& \cdot\left(\alpha_{0}^{v} \beta^{2} g \boldsymbol{\sigma}_{t s} \cdot \boldsymbol{n}_{p s}\right) d \Gamma_{p s}^{*}
\end{aligned}
$$

from which is can be deduced that $\alpha_{0}^{\omega}=\alpha_{0}^{v} g \beta=\alpha_{0}^{\rho} g^{2}$ and consequently invariance of $\alpha_{0}^{\omega} g \beta^{-1} J_{t s}^{*}$ with respect to $\beta$ (i.e. i.e. $\alpha_{0}^{\omega} g \beta^{-1} J_{t s}^{*}=J_{p s}^{*}$ ) requires $\alpha_{0}^{\omega} \beta \omega_{t s}=\alpha_{0}^{v} g \beta^{2} \omega_{t s}=$ $\omega_{p s}$.

The standard J-integral $J_{t s}$ satisfies the relationship $J_{t s}^{*}=v_{t s}^{*} J_{t s}$ and if invariant with respect to $\beta$, then $\alpha_{0}^{\omega} J_{t s}=J_{p s}$ with $\alpha_{0}^{\omega}=\alpha_{0}^{v} g \beta$. It is of interest to examine a relatively simple analytical example at this stage to demonstrate the scaling concepts on familiar material. Consider then a crack of length $2 a_{t s}$ in an infinite plate, where in this case the J-integral in the absence of plasticity has a simple analytical solution, which is

$$
J_{t s}=\frac{K_{I t s}^{2}}{E_{t s}^{\prime}}=\pi a_{t s} \frac{\sigma_{t s}^{2}}{E_{t s}^{\prime}}
$$

where stress intensity $K_{I t s}=\sigma_{t s} \sqrt{\pi a_{t s}}$ and $E_{t s}^{\prime}$ represent Young's modulus $E_{t s}$ or $E_{t s} /\left(1-v_{t s}^{2}\right)$, and where $v_{t s}$ is Poisson's ratio.

Consider then $\alpha_{0}^{\omega} J_{t s}$, which provides

$$
\alpha_{0}^{\omega} J_{t s}=\alpha_{0}^{v} g \beta J_{t s}=\pi\left(\beta^{-1} a_{t s}\right) \frac{\left(\alpha_{0}^{v} \beta^{2} g \sigma_{t s}\right)^{2}}{\alpha_{0}^{v} \beta^{2} g E_{t s}^{\prime}}
$$

where for zeroth-order conditions (e.g. $\alpha_{0}^{v} \beta^{2} g E_{t s}^{\prime}=E_{p s}^{\prime}$ ) it is evident that $\alpha_{01}^{\omega} J_{t s}\left(\beta_{1}\right)=J_{p s}$ and consequently a single trial-space experiment is sufficient.

However, observe that $\alpha_{0}^{\omega} \sqrt{\beta} K_{I t s}=\left(\alpha_{0}^{v} \beta^{2} g \sigma_{t s}\right) \sqrt{\pi \beta^{-1} a_{t s}}$ and should zeroth-order apply, then $\alpha_{0}^{\omega} \sqrt{\beta} K_{I t s}=K_{I p s}$ but it is relatively easy to break zeroth-order conditions. Using the same material for a single-scaled experiment would be suffice since $\alpha_{0}^{v}$ is insufficient for the matching of the three material properties, i.e. $K_{I c t s}=K_{I c p s}$ (fracture toughness), $E_{t s}=E_{p s}$ and $v_{t s}=v_{p s}$ (and additionally yield stress if plasticity is involved). Additional flexibility is required, and first-order finite similitude provides greater scope for capturing the full-scale physical behaviour.

\subsubsection{Analytical calculation of J}


It is of interest to examine the situation of two scale experiments were the objective is the prediction of the onset of crack propagation in an infinite full-scale plate. The situation is depicted in Fig. 5 where the possibility of using three different materials is considered for the scale choices of $\beta_{1}=\frac{1}{2}$ and $\beta_{2}=\frac{1}{4}$, and the full-scale plate. The general concept presented in Fig. 3 is recreated in Fig. 5 for the specific case of a single crack subjected to a uniform stress field, where it is assumed here that the analytical result in Eq. (18) applies to the real scaled experiments. The test for success is how close the virtual model matches the full-scale real result, which again is assumed to comply with Eq. (18). Observe from Eq. (14c) and Fig. 5 that the products $\alpha_{01}^{v} g_{1}$ and $\alpha_{02}^{v} g_{2}$ appear, which confirms that $g_{1}$ and $g_{2}$ have no part to play in what is after all a quasi-static analysis. Changing $g_{2}$ (say) can be negated by changing $\alpha_{02}^{v}$ so that the product $\alpha_{02}^{v} g_{2}$ remains unchanged. As far as the selection of $\alpha_{01}^{v}$ and $\alpha_{02}^{v}$ is concerned, these are set to satisfy zeroth-order conditions for fracture toughness being zeroth-order scalars and consequently satisfy

$$
\begin{aligned}
\boldsymbol{\sigma}_{p s}^{c r i t} & =\frac{K_{I c p s}}{\sqrt{\pi a_{p s}}}=\alpha_{01}^{v} g_{1} \beta_{1}^{2} \boldsymbol{\sigma}_{t s}^{c r i t}\left(\beta_{1}\right)=\alpha_{01}^{v} g_{1} \beta_{1}^{2} \frac{K_{I c t s}\left(\beta_{1}\right)}{\sqrt{\pi a_{t s 1}}} \\
& =\alpha_{02}^{v} g_{2} \beta_{2}^{2} \boldsymbol{\sigma}_{t s}^{c r i t}\left(\beta_{2}\right)=\alpha_{02}^{v} g_{2} \beta_{2}^{2} \frac{K_{I c t s}\left(\beta_{2}\right)}{\sqrt{\pi a_{t s 2}}}
\end{aligned}
$$

with $a_{t s 1}=a_{t s}\left(\beta_{1}\right)=\beta_{1} a_{p s}$ and $a_{t s 2}=a_{t s}\left(\beta_{2}\right)=\beta_{2} a_{p s}$ and consequently

$$
\begin{aligned}
& \alpha_{01}^{v} g_{1}=\frac{\sqrt{\beta_{1}}}{\beta_{1}^{2}} \frac{K_{\text {Ic } p s}}{K_{\text {Ic ts }}\left(\beta_{1}\right)} \\
& \alpha_{02}^{v} g_{2}=\frac{\sqrt{\beta_{2}}}{\beta_{2}^{2}} \frac{K_{\text {Ic } p s}}{K_{\text {Ic ts }}\left(\beta_{2}\right)}
\end{aligned}
$$




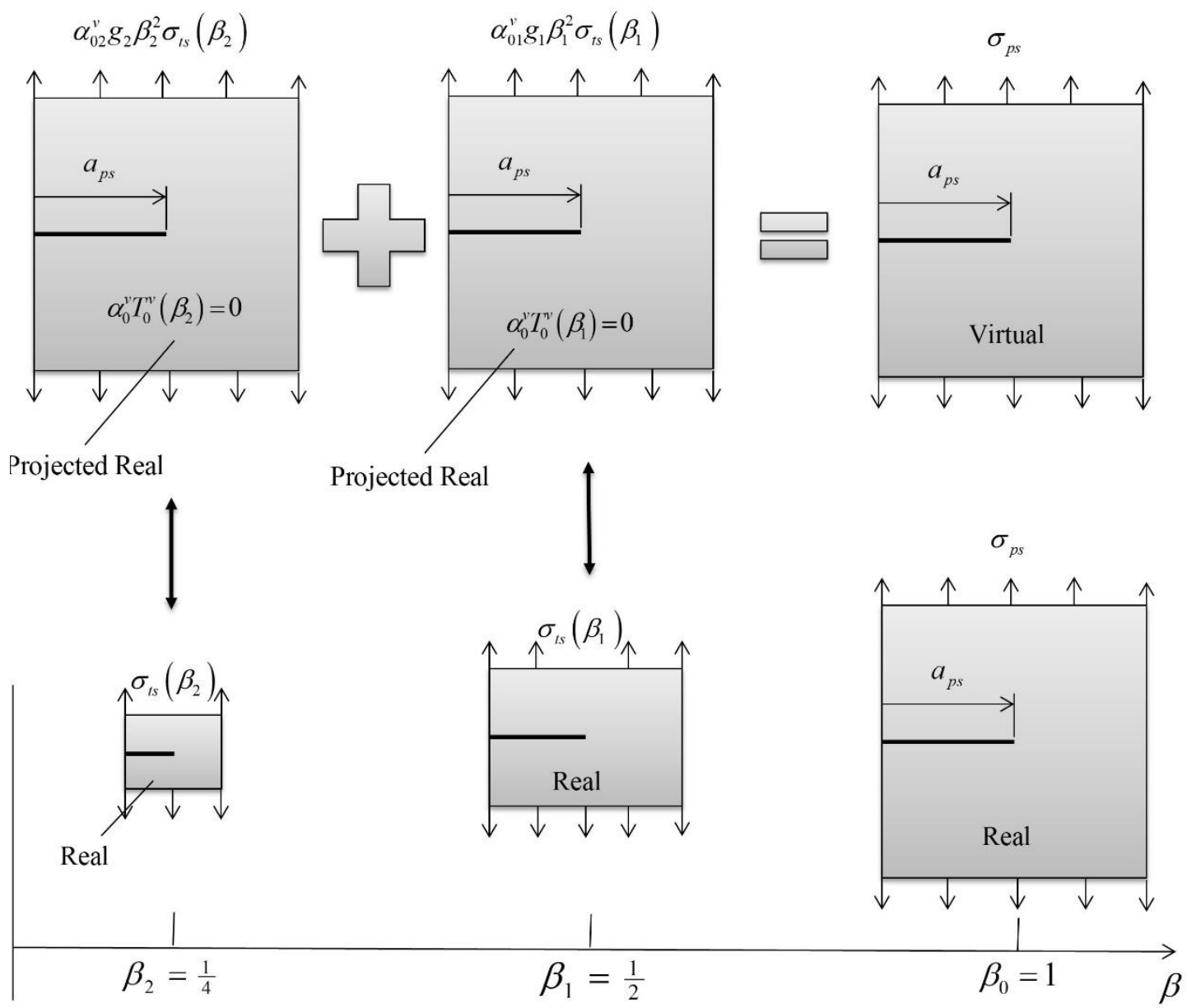

Figure 5. Portrayal of a side crack in an infinite plate subjected to a uniform stress field.

Note here that the zeroth-order conditions $\alpha_{01}^{\rho} \rho_{t s 1} \beta_{1}^{3}=\rho_{p s}$ and $\alpha_{02}^{\rho} \rho_{t s 2} \beta_{2}^{3}=\rho_{p s}$ along with $\alpha_{01}^{v}=\alpha_{01}^{\rho} g_{1} \beta_{1}^{-1}$ and $\alpha_{02}^{v}=\alpha_{02}^{\rho} g_{2} \beta_{2}^{-1}$ in Table 1 are not applied since density is not a feature of this simple problem. However, their inclusion has the not too unexpected consequence that time plays a part in the analysis. This follows because their incorporation constrains the values of $\alpha_{01}^{v}$ and $\alpha_{02}^{v}$ but Eq. (21) can still be satisfied provided $g_{1}$ and $g_{2}$ are free to be set. Observe that $\alpha_{01}^{v} g_{1}=\alpha_{01}^{\rho} g_{1}^{2} \beta_{1}^{-1}$ and $\alpha_{02}^{v} g_{1}=\alpha_{02}^{\rho} g_{2}^{2} \beta_{2}^{-1}$ and consequently on setting $g_{1}$ and $g_{2}$ equal to

$$
\begin{aligned}
& g_{1}=\sqrt{\frac{\beta_{1}^{2} \sqrt{\beta_{1}}}{\alpha_{01}^{\rho} \beta_{1}^{3}} \frac{K_{I c p s}}{K_{I c t s}}}=\beta_{1} \sqrt{\sqrt{\beta_{1}} \frac{\rho_{t s 1}}{\rho_{p s}} \frac{K_{\text {Ic } p s}}{K_{I c t s 1}}} \\
& g_{2}=\sqrt{\frac{\beta_{2}^{2} \sqrt{\beta_{2}}}{\alpha_{02}^{v} \beta_{2}^{3}} \frac{K_{I c p s}}{K_{I c t s}}}=\beta_{2} \sqrt{\sqrt{\beta_{2}} \frac{\rho_{t s 2}}{\rho_{p s}} \frac{K_{I c p s}}{K_{I c t s}}}
\end{aligned}
$$

ensures that Eqs. (21) are satisfied. 
It is understood that although time is not a feature of quasi-static processes, it is of course a feature of real experiments. Here $g_{1}$ and $g_{2}$ informs on how the information from the two experiments are combined. Although inertia is not a feature of the quasi-static process and consequently material density is not involved necessarily it can still be incorporated on imagining the loading process to occur over a specified period. This period can be different at the two scales as indicated by $g_{1} \neq g_{2}$.

It is evident from Eq. (18) that in order to be able to predict the J-integral at full scale by means of scaled experimentation it is necessary to say something about deformation and strain energy, reflected by the presence of Young's modulus in this equation. One approach is depicted in Fig. 6, which is essentially the situation in Fig. 5, with the cracks removed and the scales $\beta_{1}$ and $\beta_{2}$ assumed free.

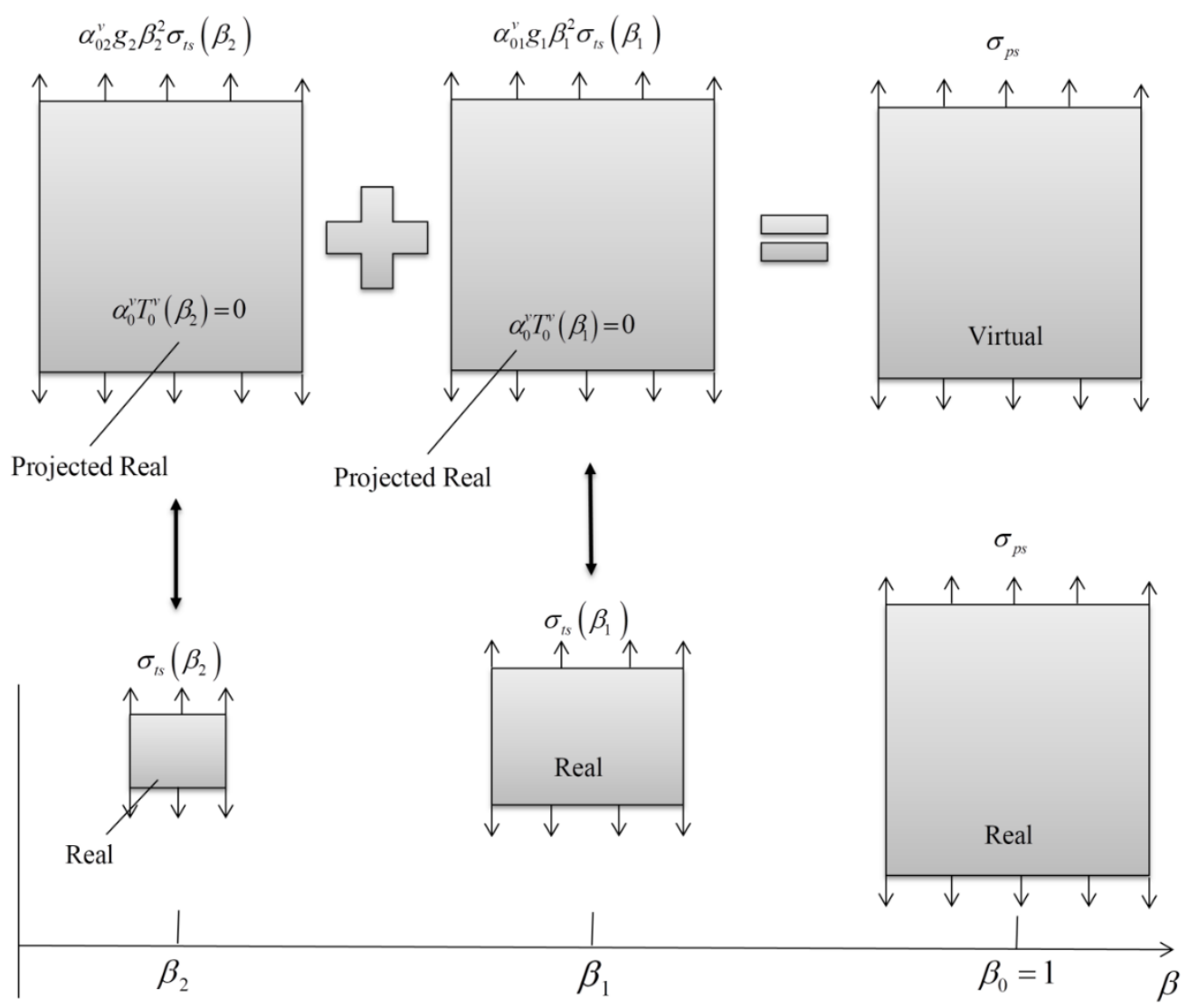

Figure 6. Imagined stress loading in the absence of a crack.

In this case at $\boldsymbol{\sigma}_{p s}=\boldsymbol{\sigma}_{p s}^{\text {crit }}$ the following zeroth-order conditions are assumed to apply

$\boldsymbol{\sigma}_{p s}^{\text {crit }}=E_{p s}^{\prime} \varepsilon_{p s}^{c r i t}=\alpha_{01}^{v} g_{1} \beta_{1}^{2} \boldsymbol{\sigma}_{t s 1}^{\text {crit }}=\alpha_{01}^{v} g_{1} \beta_{1}^{2} E_{t s 1}^{\prime} \varepsilon_{t s 1}^{c r i t}=\alpha_{02}^{v} g_{2} \beta_{2}^{2} \boldsymbol{\sigma}_{t s 2}^{\text {crit }}=$ $\alpha_{02}^{v} g_{2} \beta_{2}^{2} E_{p s 2}^{\prime} \varepsilon_{t s 2}^{c r i t}$ 
where it is assumed further that $\varepsilon_{p s}^{c r i t}=\varepsilon_{t s 1}^{c r i t}=\varepsilon_{t s 2}^{c r i t}$ and given that the conditions in Eqs. (21) apply it follows that

$$
\begin{aligned}
& \alpha_{01}^{v} g_{1}=\frac{\sqrt{\beta_{1}}}{\beta_{1}^{2}} \frac{K_{I c p s}}{K_{I c t s 1}}=\frac{1}{\beta_{1}^{2}} \frac{E_{p s}^{\prime}}{E_{t s 1}^{\prime}} \\
& \alpha_{02}^{v} g_{2}=\frac{\sqrt{\beta_{2}}}{\beta_{2}^{2}} \frac{K_{I c p s}}{K_{I c t s 1}}=\frac{1}{\beta_{2}^{2}} \frac{E_{p s}^{\prime}}{E_{t s 2}^{\prime}}
\end{aligned}
$$

which leads to specific values of $\beta_{1}$ and $\beta_{2}$ depending on the material chosen, i.e. it provides

$$
\begin{aligned}
& \beta_{1}=\left(\frac{K_{I c t s 1}}{K_{I c p s}} \frac{E_{p s}^{\prime}}{E_{t s 1}^{\prime}}\right)^{2} \\
& \beta_{2}=\left(\frac{K_{I c t s 2}}{K_{I c p s}} \frac{E_{p s}^{\prime}}{E_{t s 2}^{\prime}}\right)^{2}
\end{aligned}
$$

and since $\beta_{2} \leq \beta_{1}$ these relationships require materials that satisfy $\frac{K_{I c t s 1}}{E_{t s 1}^{\prime}} \leq \frac{K_{I c t s 2}}{E_{t s 2}^{\prime}}$.

Thus, with the extra flexibility afforded the approach by allowing $\beta_{1}$ and $\beta_{2}$ to vary, zerothorder matching is possible. It is of interest therefore to test a simple example consisting of three materials to represent the two trial and full-scale experiments. The material properties for all the materials applied in this paper are tabulated in Table 3. Specifically, the materials considered are: titanium (Ti6Al4V) [37-39], stainless steel (AISI 201), tungsten [40], steel (EN3B), copper (C101) and aluminium (6082). The three materials selected here are given in Table 4 and consist of titanium, stainless and tungsten for full and trial space at scales at $\beta_{1}$ and $\beta_{2}$, respectively. To identify the materials in the virtual models the notation Ti-Ti, Ti-SS, Ti-Tung and Ti-SS-Tung is employed to signify that the full-scale material is titanium and the scaled materials are titanium, stainless or tungsten with Ti-SS-Tung signifying that first-order theory is employed with two trial-space experiments. Eq. (18) is applied to determine the Jintegral for the virtual model, with length scales set by Eqs. (25) and $\alpha_{01}^{v} g_{1}$ and $\alpha_{02}^{v} g_{2}$ are calculated using Eqs. (24). As alluded to above however, this arrangement ensures that zeroth-order theory is sufficient and consequently $J_{p s}=\alpha_{01}^{v} g_{1} \beta_{1} J_{t s 1}$ and $\alpha_{01}^{v} g_{1} \beta_{1} J_{t s 1}=$ $\alpha_{02}^{v} g_{2} \beta_{2} J_{t s 2}$. The objective here is to predict the J-integral (at its critical value) at full scale which is known to satisfy Eq. (18). Examination of Table 4 confirms that the three combinations Ti-Ti, Ti-SS, Ti-Tung gives as expected the exact prediction for both $E_{p s}$ and $J_{c p s}$, i.e. $120 \mathrm{GPa}$ and $100.8 \mathrm{~kJ} / \mathrm{m}^{2}$, respectively, as tabulated for titanium in the first row of Table 3. 
Table 3. Materials properties used in the paper.

\begin{tabular}{|c|c|c|c|c|c|c|}
\hline Material & $\begin{array}{c}E \\
(\mathrm{GPa})\end{array}$ & $\begin{array}{c}\sigma_{Y} \\
(\mathrm{MPa})\end{array}$ & $\begin{array}{c}\sigma_{T S} \\
(\mathrm{MPa})\end{array}$ & $\begin{array}{c}K_{I C} \\
\left(\mathrm{GPa} \mathrm{m}^{\frac{1}{2}}\right)\end{array}$ & $\begin{array}{c}G_{C} \\
\left(\mathrm{~kJ} / \mathrm{m}^{2}\right)\end{array}$ & $\begin{array}{c}v \\
(\text { Poisson } \\
\text { ratio })\end{array}$ \\
\hline Titanium & 120 & 812 & 1077 & 1100 & 100.8 & 0.36 \\
\hline Stainless & 200 & 881 & 1206 & 100 & 50.0 & 0.30 \\
\hline Steel & 190 & 324 & 491 & 50 & 13.2 & 0.29 \\
\hline Tungsten & 410 & 1123 & 1255 & 150 & 54.9 & 0.28 \\
\hline Copper & 130 & 92 & 243 & 30 & 11.7 & 0.34 \\
\hline Aluminium & 70 & 98 & 370 & 30 & 12.9 & 0.35 \\
\hline
\end{tabular}

Table 4. Virtual material properties for three material combination.

\begin{tabular}{|c|c|c|c|c|c|c|c|}
\hline \multirow[t]{2}{*}{$\mathrm{i}$} & \multirow[t]{2}{*}{ Material } & \multirow[t]{2}{*}{$\beta_{i}$} & \multirow{2}{*}{$\begin{array}{l}J_{c}=\frac{K_{I c}^{2}}{E} \\
\left(\mathrm{~kJ} / \mathrm{m}^{2}\right)\end{array}$} & \multirow[t]{2}{*}{$\alpha_{0 i}^{v} g_{i}$} & \multicolumn{3}{|c|}{ Virtual Properties } \\
\hline & & & & & $\begin{array}{c}E_{p s} \\
(\mathrm{GPa})\end{array}$ & $\begin{array}{c}J_{c p s} \\
\left(\mathrm{~kJ} / \mathrm{m}^{2}\right)\end{array}$ & $\begin{array}{l}\text { Mat. } \\
\text { type }\end{array}$ \\
\hline 0 & $\begin{array}{l}\text { Titanium } \\
\text { (ps) }\end{array}$ & 1 & 100.8 & 1 & 120 & 100.8 & Ti-Ti \\
\hline 1 & $\begin{array}{l}\text { Stainless } \\
\text { (ts) }\end{array}$ & 0.2975 & 50.0 & 6.78 & 120 & 100.8 & Ti-SS \\
\hline 2 & $\begin{array}{c}\text { Tungsten } \\
\text { (ts) }\end{array}$ & 0.1593 & 54.9 & 11.53 & 120 & 100.8 & Ti-Tung \\
\hline
\end{tabular}

As a further check on the analytical study each of the three CT specimens at the length scales and materials specified in Table. 2 are re-evaluated numerically with the commercial code Abaqus. This involved meshing three CT specimens that are scaled versions of the model depicted in Fig. 4. The top circle depicted in Fig. 4 is displacement by $0.25 \mathrm{~mm}$ in the physical space (to ensure elasticity) with the bottom circle is fixed in all directions. The Jintegrals in this instance were determined using the facility within Abaqus and the results are provided in Table 5.

Table 5. Numerical J-integral comparison between the virtual and numerical models.

\begin{tabular}{|c|c|c|c|c|c|}
\hline $\mathrm{i}$ & Material & $\beta_{i}$ & $J_{t s}\left(\beta_{i}\right)$ & $\alpha_{0 i}^{v} g_{i}$ & Virtual \\
\hline
\end{tabular}




\begin{tabular}{|c|c|c|c|c|c|c|c|}
\hline & & $\left(\mathrm{kJ} / \mathrm{m}^{2}\right)$ & & $\begin{array}{c}E_{p s} \\
(\mathrm{GPa})\end{array}$ & $\begin{array}{c}J_{p s} \\
\left(\mathrm{~kJ} / \mathrm{m}^{2}\right)\end{array}$ & $\begin{array}{c}\text { Mat. } \\
\text { type }\end{array}$ \\
\hline 0 & $\begin{array}{c}\text { Titanium } \\
(\mathrm{ps})\end{array}$ & 1 & 13.1 & 1 & 120 & 13.1 & Ti-Ti \\
\hline 1 & $\begin{array}{c}\text { Stainless } \\
(\mathrm{ts})\end{array}$ & 0.2975 & 3.2 & 6.78 & 120 & 13.1 & Ti-SS \\
\hline 2 & $\begin{array}{c}\text { Tungsten } \\
(\mathrm{ts})\end{array}$ & 0.1593 & 6.2 & 11.53 & 120 & 13.1 & Ti-Tung \\
\hline
\end{tabular}

The results confirm the veracity of the theory for predicting J-integrals with scaled tests for the CT specimens, although the analysis is limited here to linear elastic fracture mechanics. Further details on the numerical simulation are provided in subsequent sections, where elastic-plastic fracture mechanics is considered.

\subsection{Cohesive zone model}

The cohesive zone model represents damage that results in fracture by means of a single failure usually along a predefined path or along element edges in any finite element analysis. The most rudimentary cohesive model is identified by a traction-separation curve consisting of three properties, which are critical stress $\sigma_{c}$, critical separation $\delta_{c}$ and the area under the curve $G_{c}$, which is the cohesive fracture energy. In the simplest linear case $G_{c}=0.5 \sigma_{c} \delta_{c}$ and it is evident that the three properties are related. The J-integral property $J_{c}=G_{c}$ suggests that $\alpha_{0}^{\omega} G_{t s}$ should be considered for the purpose of scaling. Note that

$$
\alpha_{0}^{\omega} G_{c t s}=\alpha_{0}^{v} g \beta G_{c t s}=\frac{1}{2}\left(\alpha_{0}^{v} \beta^{2} g \sigma_{c t s}\right)\left(\beta^{-1} \delta_{c t s}\right)
$$

where it is apparent that for zeroth-order scaling to apply, critical material properties must be changed.

However, theoretically the critical separation $\delta_{c}$ would be affected by $\beta$, and ultimately stress $\sigma_{c}$ will not changed with $\beta$ for the same material, which makes $G_{c}$ affected by $\beta$ but it should not be. This provides a conflict in zeroth-order theory and leads to the need of high-order theories.

In order to gain some insight into the response of a cohesive element under scaling it is of interest to consider 1-D rod (see ref. [41]) represented by a linear spring and linear cohesive model as depicted in Fig. 7. 


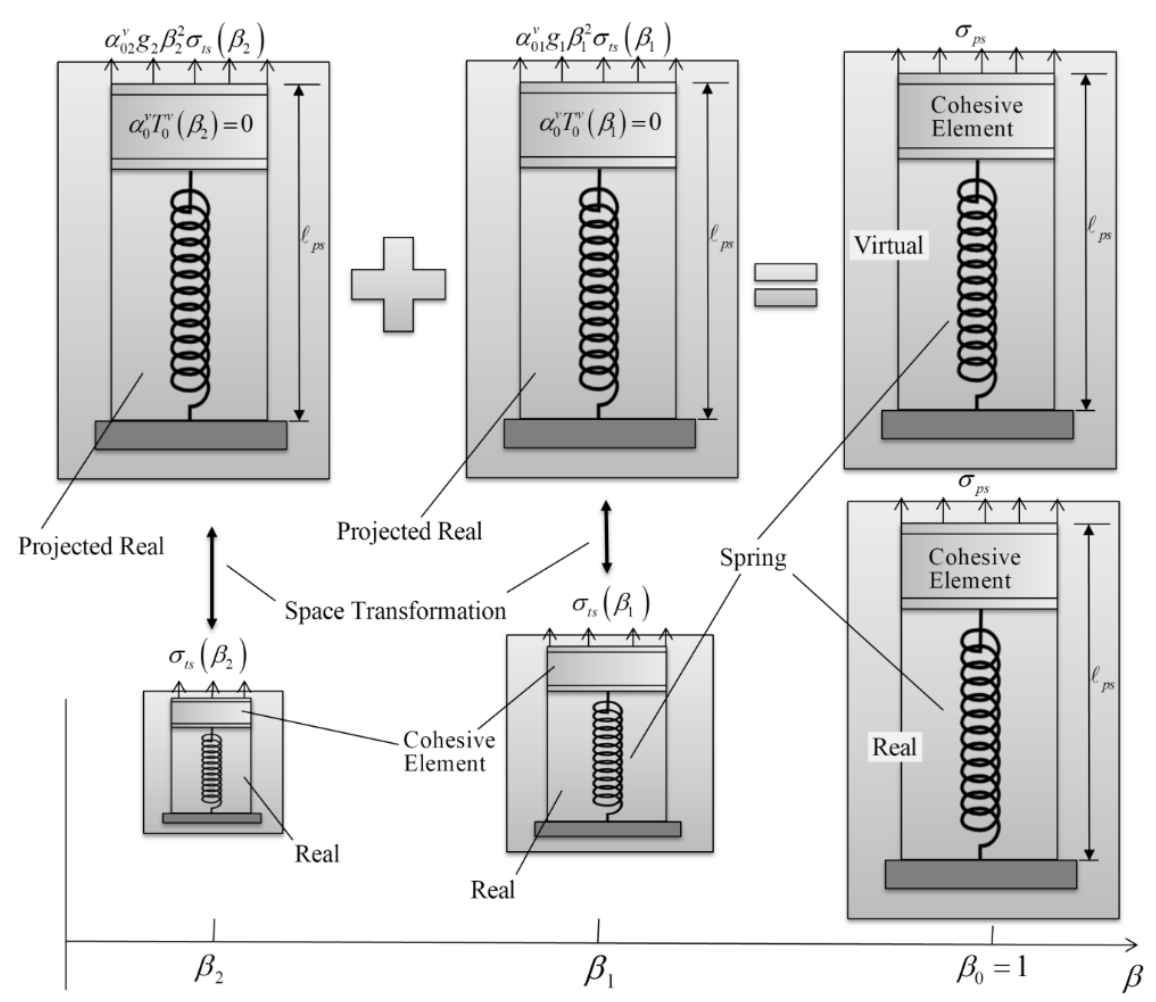

Figure 7. Scaling of rod presented by a spring and cohesive element.

Consider then a rod of length $\ell_{0}$ and area $A_{0}$ that is subjected a displacement $\delta$ at the free end with its lower end fixed. The behaviour of all the real experiments in Fig. 7 is linear extension followed by linear unloading once the critical stress $\sigma_{c}$ is attained. The total extension of the $\operatorname{rod}$ is $\delta=\delta^{e l}+\delta^{c o h}$ with uniaxial applied stress $\sigma=\sigma^{e l}=\sigma^{c o h}$, with $\delta^{e l}=\frac{\sigma^{e l} l_{0}}{E}$ and when meaningful $\sigma^{c o h}=\sigma_{c}\left(1-\frac{\delta^{c o h}}{\delta_{c}}\right)$, where $E$ is Young's modulus, $\delta^{e l}$ and $\delta^{c o h}$ are the extensions arising from the spring and cohesive element, respectively. Note that the equilibrium condition $\sigma^{e l}=\sigma^{c o h}$ provides the expression:

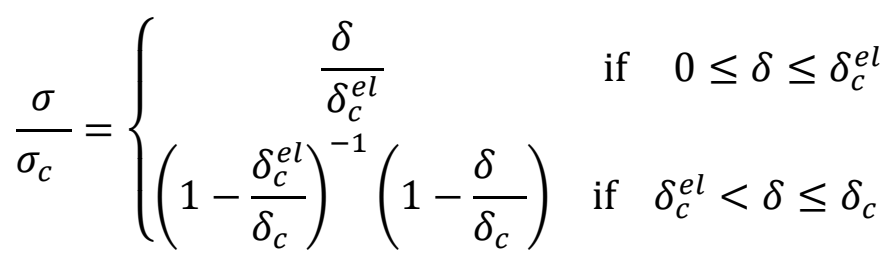

where $\delta_{c}^{e l}=\frac{\sigma_{c} \ell_{0}}{E}$ and it is assumed that this equation applies to each of the real experiments in Fig. 7; in particular $\ell_{0}$ takes up the values $\ell_{p s}, \beta_{1} \ell_{p s}$, and $\beta_{2} \ell_{p s}$ for each of the lengths of the rods.

The focus of interest is the behaviour of the full-scale virtual model depicted in Fig. 8. The scaling theory does not provide explicit function relationships of the type in Eq. (27) as it 
supplies both the stress $\sigma_{p s}$ and the displacement $u_{p s}=\delta_{p s}$ by means of Eqs. (14c) and (14e), respectively. The issue alluded to above with regards to $G_{c}$ being dependent on $\beta$ is made explicit here since with $\sigma_{c}$ and $E$ fixed for the same material, then $\delta_{c}^{e l}=\frac{\sigma_{c}}{E} \ell_{0}$ will scale with the specimen size and consequently, so must $\delta$ and $\delta_{c}$ for Eq. (27) to remain unchanged. Unfortunately, this provides a contradiction since $G_{c}=0.5 \sigma_{c} \delta_{c}$, which means $G_{c}$ scales with length also. As this is not the case $\sigma_{c}$ and $\delta_{c}$ must be allowed to vary (for zerothorder finite similitude) and this aspect in many respects highlights the problem faced in the literature with setting $\sigma_{c}$ (or $\delta_{c}$ ) with these types of element. Thus for zeroth-order scaling with $\sigma_{p s}=\alpha_{01}^{v} g_{1} \beta_{1}^{2} \sigma_{t s 1}$ and $u_{p s}=\beta_{1}^{-1} u_{t s 1}$ it is clear that with all displacement and stress terms in Eq. (27) behaving like $\delta_{p s}=\beta_{1}^{-1} \delta_{t s 1}$ and $\sigma_{p s}=\beta_{1} \sigma_{t s 1}\left(\right.$ i.e. $\alpha_{01}^{v} g_{1}=\beta_{1}^{-1}$ ), then $G_{c t s}=G_{c p s}$ and successful zeroth-order scaling is possible.

It is interest to examine two different materials for trial space models and two cases are considered (i) $G_{c t s 1}=G_{c t s 2}, \beta_{1} E_{c t s 1}=\beta_{2} E_{c t s 2}$ and (ii) $\beta_{1}^{-1} G_{c t s 1}=\beta_{2}^{-1} G_{c t s 2}, E_{c t s 1}=$ $E_{c t s 2}$; in both cases it is assumed $\beta_{1}=\frac{1}{2}$ and $\beta_{2}=\frac{1}{4}$. The zeroth-order condition $\alpha_{01}^{v} g_{1} \beta_{1}^{2} E_{t s 1}=\alpha_{02}^{v} g_{2} \beta_{2}^{2} E_{t s 2}$ is satisfied in (i) with $\alpha_{01}^{v} g_{1}=\beta_{1}^{-1}$ and $\alpha_{02}^{v} g_{2}=\beta_{2}^{-1}$ and (ii) with $\alpha_{01}^{v} g_{1}=\beta_{1}^{-2}$ and $\alpha_{02}^{v} g_{2}=\beta_{2}^{-2}$. The parameters for the two cases are tabulated in Table 6 in dimensionless form and the arrangements are depicted in Fig. 8. Examination of the figure reveals how the real behaviours are projected and then combined to form virtual models. In this case, to keep things reasonably simple, the arrangement is confined to zerothorder constructs with more complex problems (involving plasticity) being presented in the following section.

Table 6. Test parameters for scaled cohesive elements

\begin{tabular}{|c|c|c|c|c|c|c|}
\hline Test Case & $\frac{G_{c t s 1}}{G_{c t s 2}}$ & $\frac{E_{t s 1}}{E_{t s 2}}$ & $\frac{\sigma_{c t s 1}}{\sigma_{c t s 2}}$ & $\frac{\delta_{c t s 1}}{\delta_{c t s 2}}$ & $\frac{\ell_{t s 1}}{\ell_{t s 2}}$ & $\frac{\delta_{c t s 1}^{e l}}{\delta_{c t s 2}^{e l}}$ \\
\hline (i) & 1 & 0.5 & 0.5 & 2 & 2 & 2 \\
\hline (ii) & 2 & 1 & 1 & 2 & 2 & 2 \\
\hline
\end{tabular}




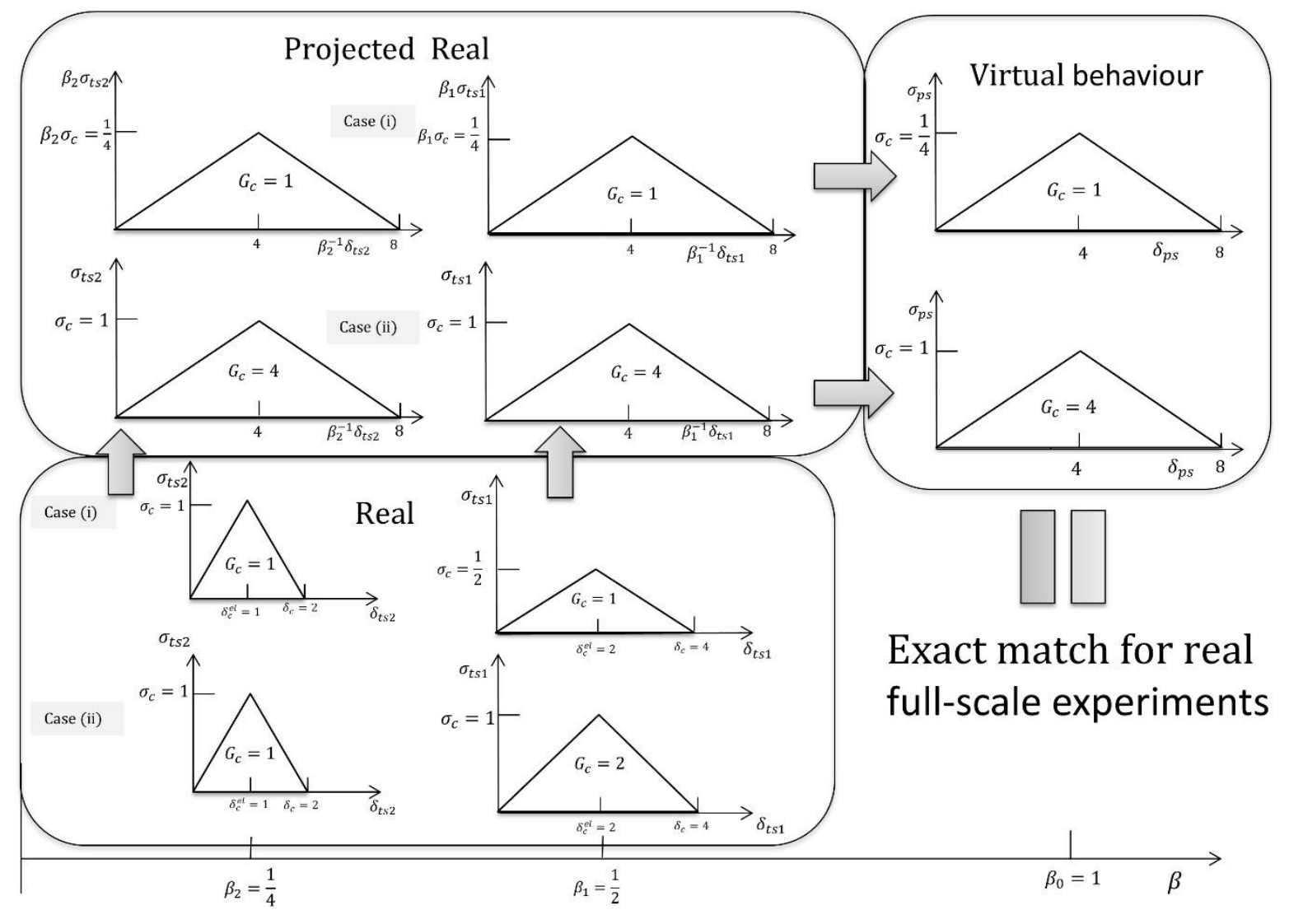

Figure 8 . The construction of virtual cohesive models for two test-case materials

\section{Non-linear material selection}

All the examples considered above are for linear materials and it is of interest to involve plasticity for physical modelling and examine how scaling parameters are set. Stress and strain are the principal focus here and the solution of

$$
\begin{gathered}
\boldsymbol{\sigma}_{p s}=\alpha_{01}^{v} g_{1} \beta_{1}^{2} \boldsymbol{\sigma}_{t s 1}+R_{1}\left(\alpha_{01}^{v} g_{1} \beta_{1}^{2} \boldsymbol{\sigma}_{t s 1}-\alpha_{02}^{v} g_{2} \beta_{2}^{2} \boldsymbol{\sigma}_{t s 2}\right) \\
\boldsymbol{\varepsilon}_{p s}=\boldsymbol{\varepsilon}_{t s 1}+R_{1}\left(\boldsymbol{\varepsilon}_{t s 1}-\boldsymbol{\varepsilon}_{t s 2}\right)
\end{gathered}
$$

which are Eqs. (14c) and (15) reproduced here for readability sake.

The scaling parameters $\alpha_{01}^{v}$ and $\alpha_{02}^{v}$ are set here based on zeroth-order conditions followed subsequently with the setting of $R_{1}$. The way this is achieved is to target critical material properties in the physical space and consider a scenario where Eqs. (28) can be set. To aid in this analysis it is useful first to reduce Eqs. (28), which are tensorial relationships, into scalar equations. This is achieved here with a proportional-fields concept, where the following relationships are assumed: 


$$
\begin{aligned}
\boldsymbol{\sigma}_{t s 1} & =\hat{a}_{1} \boldsymbol{\sigma}_{p s} \\
\boldsymbol{\sigma}_{t s 2} & =\hat{a}_{2} \boldsymbol{\sigma}_{p s} \\
\boldsymbol{\varepsilon}_{t s 1} & =\hat{b}_{1} \boldsymbol{\varepsilon}_{p s} \\
\boldsymbol{\varepsilon}_{t s 2} & =\hat{b}_{2} \boldsymbol{\varepsilon}_{p s}
\end{aligned}
$$

which on substitution into Eqs. (28) provide

$$
\begin{gathered}
1=\alpha_{01}^{v} g_{1} \beta_{1}^{2} \hat{a}_{1}+R_{1}\left(\alpha_{01}^{v} g_{1} \beta_{1}^{2} \hat{a}_{1}-\alpha_{02}^{v} g_{2} \beta_{2}^{2} \hat{a}_{2}\right) \\
1=\hat{b}_{1}+R_{1}\left(\hat{b}_{1}-\hat{b}_{2}\right)
\end{gathered}
$$

which are the sought scalar equations, where $\hat{a}_{1}, \hat{a}_{2}, \hat{b}_{1}$ and $\hat{b}_{2}$ are dimensionless parameters. It is important to appreciate here that the assumed proportional form of Eqs. (29) does not constrain the governing fields to be proportional since the purpose of Eqs. (30) is limited to the determination of scaling parameters. The determination of these dimensional parameters transpires to be relatively straightforward. In the case of zeroth-order theory with one trial experiment a simple process would be to consider synchronised uniaxial tests in both the trial space and physical space. At the point when both bars reach yield stresses $Y_{t s 1}, Y_{p s}$ and yield strains $\varepsilon_{t s 1}^{Y}=\frac{Y_{t s 1}}{E_{t s 1}}, \varepsilon_{p s}^{Y}=\frac{Y_{p s}}{E_{p s}}$, then Eqs. (30) reduce to $1=\alpha_{01}^{v} g_{1} \beta_{1}^{2} \hat{a}_{1}$ and $1=\hat{b}_{1}$ with $\hat{a}_{1}=$ $\frac{Y_{t s 1}}{Y_{p s}}$ and $\hat{b}_{1}=\frac{\varepsilon_{t s 1}^{Y}}{\varepsilon_{p s}^{Y}}$, which provides the means to set $\alpha_{01}^{v} g_{1}$, ie $\alpha_{01}^{v} g_{1} \beta_{1}^{2}=\frac{1}{\hat{a}_{1}}=\frac{Y_{p s}}{Y_{t s 1}}$. Strains match, for zeroth-order finite similitude, which is precisely what is inferred by the equation $\hat{b}_{1}=1$ or equivalently $\varepsilon_{t s 1}^{Y}=\varepsilon_{p s}^{Y}$, which gives $\frac{Y_{t s 1}}{E_{t s 1}}=\frac{Y_{p s}}{E_{p s}}$ or $E_{p s}=E_{t s 1} \frac{Y_{p s}}{Y_{t s 1}}$. As regards first order, the zeroth-order conditions $\hat{a}_{1}=\frac{Y_{t s 1}}{Y_{p s}}, \hat{a}_{2}=\frac{Y_{t s 2}}{Y_{p s}}, 1=\alpha_{01}^{v} g_{1} \beta_{1}^{2} \hat{a}_{1}$ and $1=\alpha_{02}^{v} g_{2} \beta_{2}^{2} \hat{a}_{2}$ are assumed to apply making $\alpha_{02}^{v} g_{2} \beta_{2}^{2}$ and $\alpha_{01}^{v} g_{1} \beta_{1}^{2}$ known. To determine $R_{1}$, Eq. (30a) is reused, where in this case mean values for stress are utilised. This approach enables the effect of material work hardening to be accommodated and the method was first introduced in reference [34] although limited to zeroth-order problems in that case. The mean-value is defined to be

$$
\bar{\sigma}=\frac{1}{\varepsilon^{\max }} \int_{0}^{\varepsilon^{\max }} \sigma d \varepsilon
$$

where $\varepsilon^{\max }$ is taken from the material stress-strain curves used in the experiments; these are depicted in Fig. 9 with is $\varepsilon^{\max }$ indicated for the materials introduced in Table 3. Note from 
the figure that $\varepsilon^{\max }$ is limited to 0.1 for materials with strain ranges greater than that for titanium.

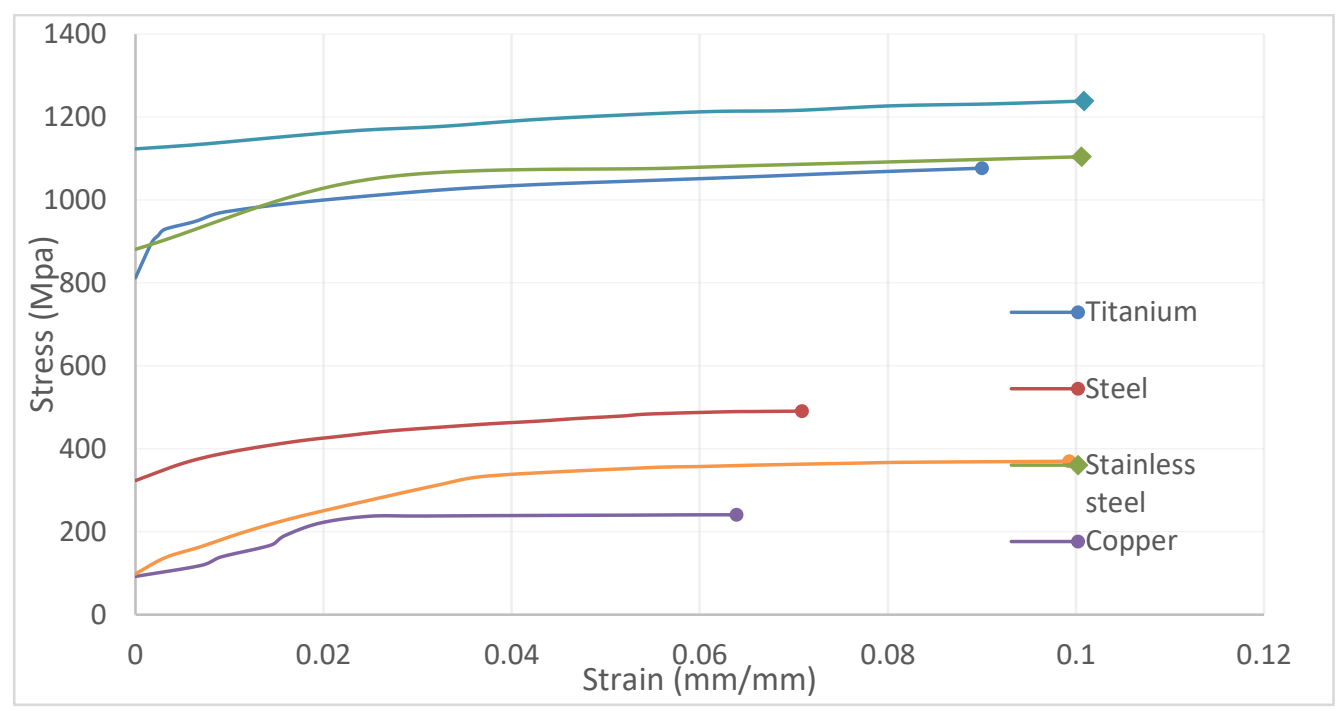

Figure 9. Strain-hardening curves for materials listed in Table 1.

With the application of Eq. (31) it follows that Eq. (30a) can be rearranged to give

$$
R_{1}=\frac{1-\alpha_{01}^{v} g_{1} \beta_{1}^{2} \hat{a}_{1}}{\alpha_{01}^{v} g_{1} \beta_{1}^{2} \hat{a}_{1}-\alpha_{02}^{v} g_{2} \beta_{2}^{2} \hat{a}_{2}}
$$

where in this case $\hat{a}_{1}=\frac{\bar{\sigma}_{t s 1}}{\bar{\sigma}_{p s}}, \hat{a}_{2}=\frac{\bar{\sigma}_{t s 2}}{\bar{\sigma}_{p s}}$, and where it is appreciated that this approach for the determination of the scaling parameters is one of many possibilities; no attempt is made here to determine the length scales $\beta_{1}$ and $\beta_{2}$, which are set here to be $\beta_{1}=\frac{1}{2}$ and $\beta_{2}=\frac{1}{4}$.

\subsection{Zeroth-order material selection}

The materials presented in Table 3 are utilised as trial materials with titanium being the material chosen for the physical model. The objective here is to examine how well material choices for the trial space capture the uniaxial behaviour of titanium.

Table 5. Zeroth-order virtual material properties with yield-stress targeting and $\beta_{1}=\frac{1}{2}$.

\begin{tabular}{|c|c|c|c|c|c|}
\hline Material & Yield & $\alpha_{01}^{v} g_{1}$ & Virtual & \multicolumn{2}{|c|}{ Virtual material properties } \\
stress & & Material & \multicolumn{2}{|c|}{} \\
\cline { 4 - 5 } & $(\mathrm{MPa})$ & & & Yield stress & Young's \\
& & & & \\
& & & & & Modulus E \\
& & & & & \\
\hline
\end{tabular}




\begin{tabular}{|c|c|c|c|c|c|}
\hline Titanium (PS) & 812 & 4 & Ti-Ti & 812 & 120 \\
\hline Stainless steel (TS) & 881 & 3.69 & Ti-SS & 812 & 120 \\
\hline Steel (TS) & 324 & 10.04 & Ti-S & 812 & 120 \\
\hline Tungsten (TS) & 1123 & 2.89 & Ti-Tung & 812 & 120 \\
\hline Copper (TS) & 92 & 35.25 & Ti-C & 812 & 120 \\
\hline Aluminium (TS) & 98 & 30.03 & Ti-Al & 812 & 120 \\
\hline
\end{tabular}

Recall that a virtual-material model is produced by targeting selected materials properties (e.g. yield stress) of titanium where in the case stainless steel as the trial material it is noted as Ti-SS. The scaling parameters $\alpha_{01}^{v} g_{1}$ for each of the material combinations are presented in Table 5, where it is apparent that the virtual materials match both the yield stress and Young's modulus of titanium. The stress strain curves for titanium and the virtual material can be found in Fig. 10, where large deviations are apparent for some combinations. The virtual material model Ti-SS gives the closest match with Ti-Al providing the worst. The issue here is that although yield stress and Young's modulus match, there is mismatch in strain hardening and with zeroth-order theory there is no practical solution to this dilemma.

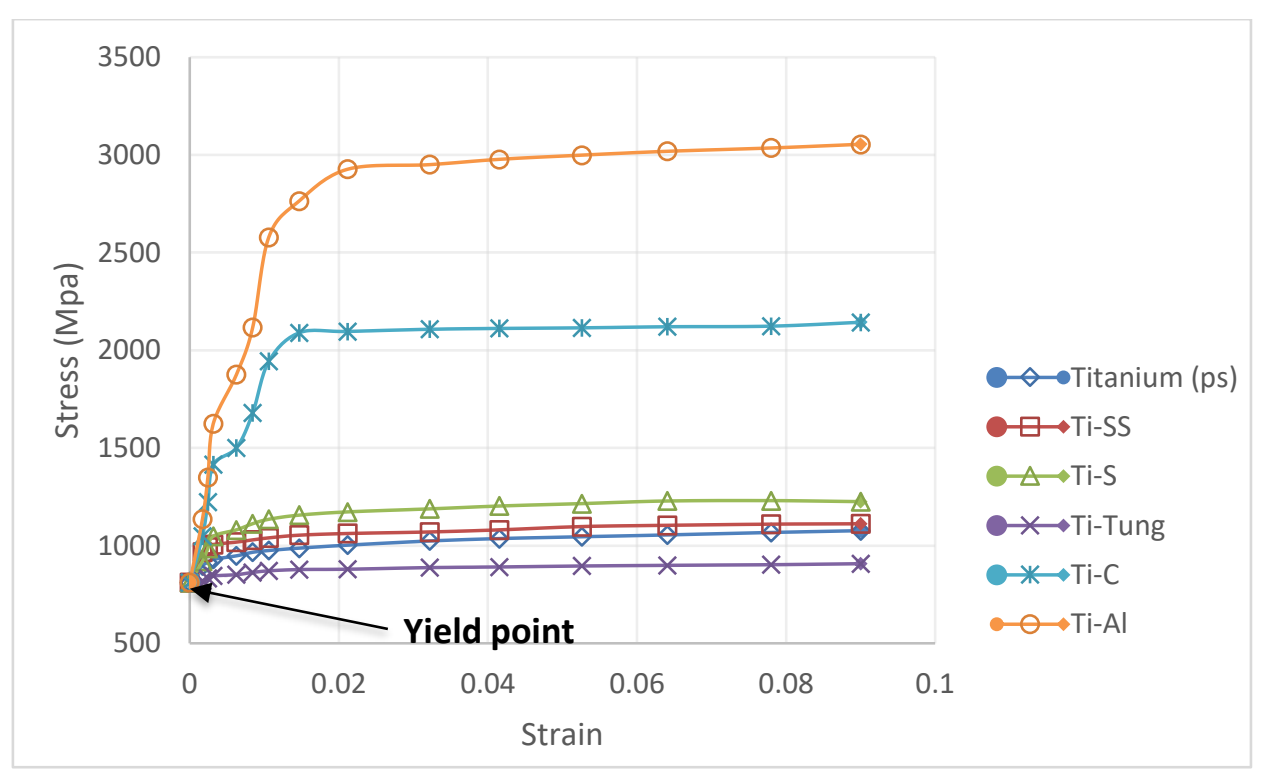

Figure 10. Stress-hardening curves for virtual models targeting titanium yield stress. 
Table 6. First-order virtual material properties with $\beta_{1}=\frac{1}{2}$. and $\beta_{2}=\frac{1}{4}$.

\begin{tabular}{|c|c|c|c|c|c|c|c|c|}
\hline \multirow[t]{2}{*}{ Material } & \multirow[t]{2}{*}{$\varepsilon_{\max }$} & \multirow{2}{*}{$\begin{array}{l}\text { Mean } \\
\text { stress } \\
(\mathrm{MPa})\end{array}$} & \multirow{2}{*}{$\begin{array}{l}\alpha_{01}^{v} g_{1} \\
\left(\beta_{1}=\frac{1}{2}\right)\end{array}$} & \multirow{2}{*}{$\begin{array}{c}\alpha_{02}^{v} g_{2} \\
\left(\beta_{2}=\frac{1}{4}\right)\end{array}$} & \multirow{2}{*}{$\begin{array}{c}\text { Virtual } \\
\text { Material }\end{array}$} & \multirow[t]{2}{*}{$R_{1}$} & \multicolumn{2}{|c|}{ Virtual properties } \\
\hline & & & & & & & $\begin{array}{l}\text { Mean } \\
\text { stress } \\
(\mathrm{MPa})\end{array}$ & $\begin{array}{c}\text { Yield } \\
\text { stress } \\
(\mathrm{MPa})\end{array}$ \\
\hline $\begin{array}{l}\text { Titanium } \\
\text { (PS) }\end{array}$ & 0.09 & 1026 & 4 & 16 & Ti-SS-S & -0.386 & 1022 & 812 \\
\hline $\begin{array}{c}\text { Stainless } \\
\text { steel (TS) }\end{array}$ & 0.10 & 1051 & 3.69 & 14.76 & Ti-SS-C & -0.647 & 1013 & 812 \\
\hline Steel (TS) & 0.07 & 445 & 10.04 & 40.16 & $\begin{array}{l}\text { Ti-SS- } \\
\text { Tung }\end{array}$ & 0.539 & 1023 & 812 \\
\hline $\begin{array}{c}\text { Tungsten } \\
\text { (TS) }\end{array}$ & 0.10 & 1194 & 2.89 & 11.56 & Ti-S-C & 0.124 & 1039 & 812 \\
\hline Copper (TS) & 0.06 & 210 & 35.25 & 141.0 & $\begin{array}{l}\text { Ti-C- } \\
\text { Tung }\end{array}$ & -0.835 & 1010 & 812 \\
\hline $\begin{array}{l}\text { Aluminium } \\
\text { (TS) }\end{array}$ & 0.10 & 312 & 30.03 & 120.12 & Ti-SS-Al & -0.035 & 1014 & 812 \\
\hline
\end{tabular}

Considering the results presented in Fig. 10 it is of interest to examine what improvement can be made on the application of first-order theory to the materials in Table 3.

\subsection{First-order material selection}

Following the procedure outlined above, both $\alpha_{01}^{v} g_{1}$ and $\alpha_{02}^{v} g_{2}$ are determined on targeting the yield stress and are tabulated in Table 6. Additionally, $R_{1}$ is evaluated based on Eq. (31) with the objective of matching the mean stress for titanium. The properties of real and virtual materials are provided in Table 6 and stress-strain curves over the strain range for titanium are depicted in Fig. 11. On contrasting Figs. (10) and (11) it is apparent just how marked the improvements are with significantly reduced disparity between the titanium and all other curves. A perfect match is not revealed but the improvement brought about by an additional trial experiment is demonstrable. 


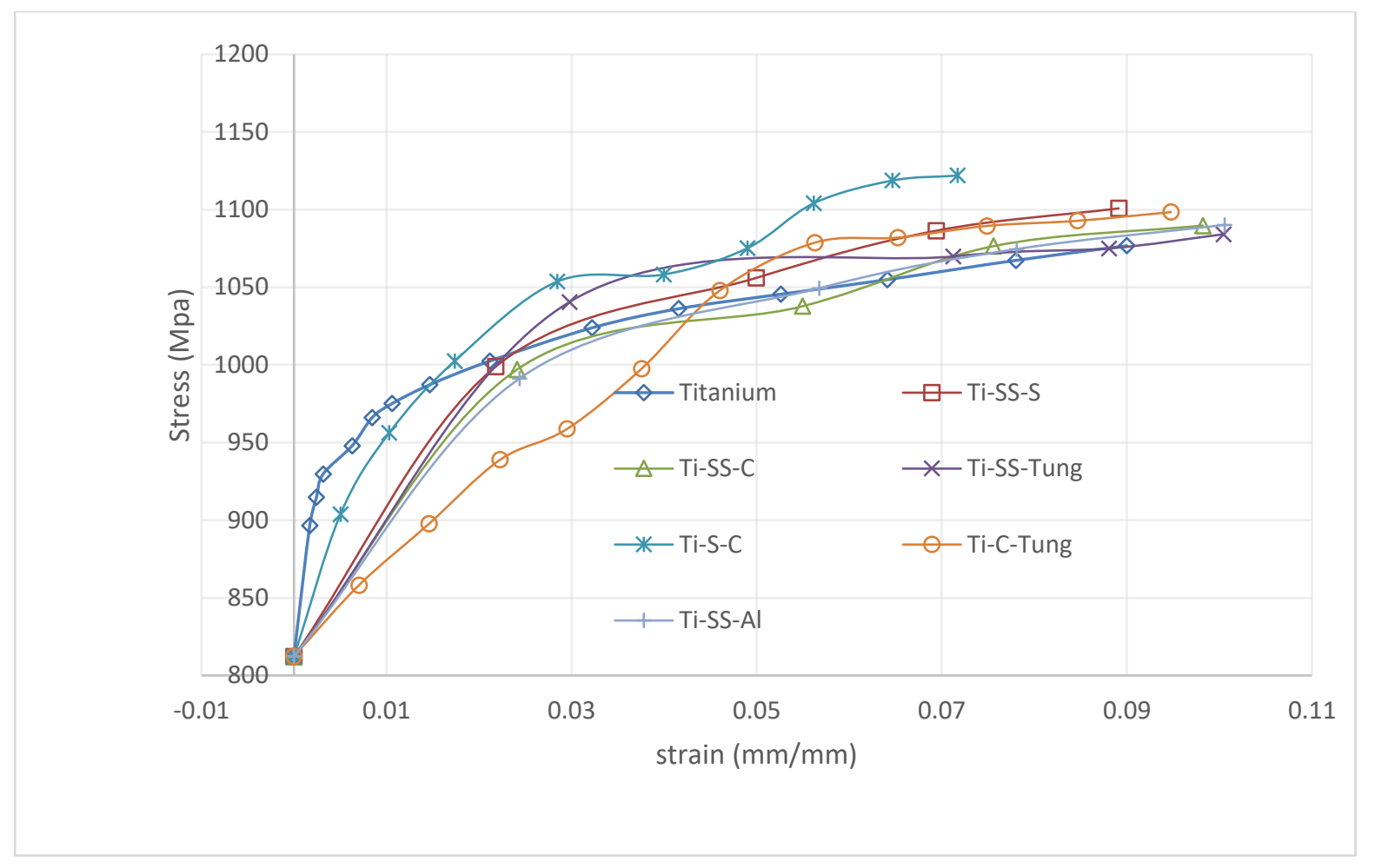

Figure 11. Strain-hardening curves for virtual materials over the strain range for titanium.

\section{Test specimen analysis (numerical)}

The virtual materials of Section 7 are tested numerically using the commercial finite element package Abaqus.[42]. The context of the study is physical modelling with a view of examining how one (zeroth order) or two (first order) modelling materials can be used to predict the behaviour of a full-scale model. In practice experimental trials would replace the numerical models but the focus here is on confirming the validity of the finite similitude approach and numerical simulation is sufficient for that purpose. Two relatively simple classical elastic-plastic fracture mechanics problems are considered; these are the quasistatically loaded 2D compact-tension (CT) specimen along with the 3D three-point bending (TPB) problem. The evaluation of the J-integral is the focus using the inbuilt facility within the Abaqus software and the evaluation of the zeroth and first-order theories.

\subsection{Compact tension and bending models}

The compact tension specimen depicted in Fig. 4 is meshed and loaded in the manner depicted in Fig. 12, where a displacement of $2 \mathrm{~mm}$ is applied to initiate plastic behaviour. The mesh consists of 4-noded continuum plane-stress elements (CPS4R). 

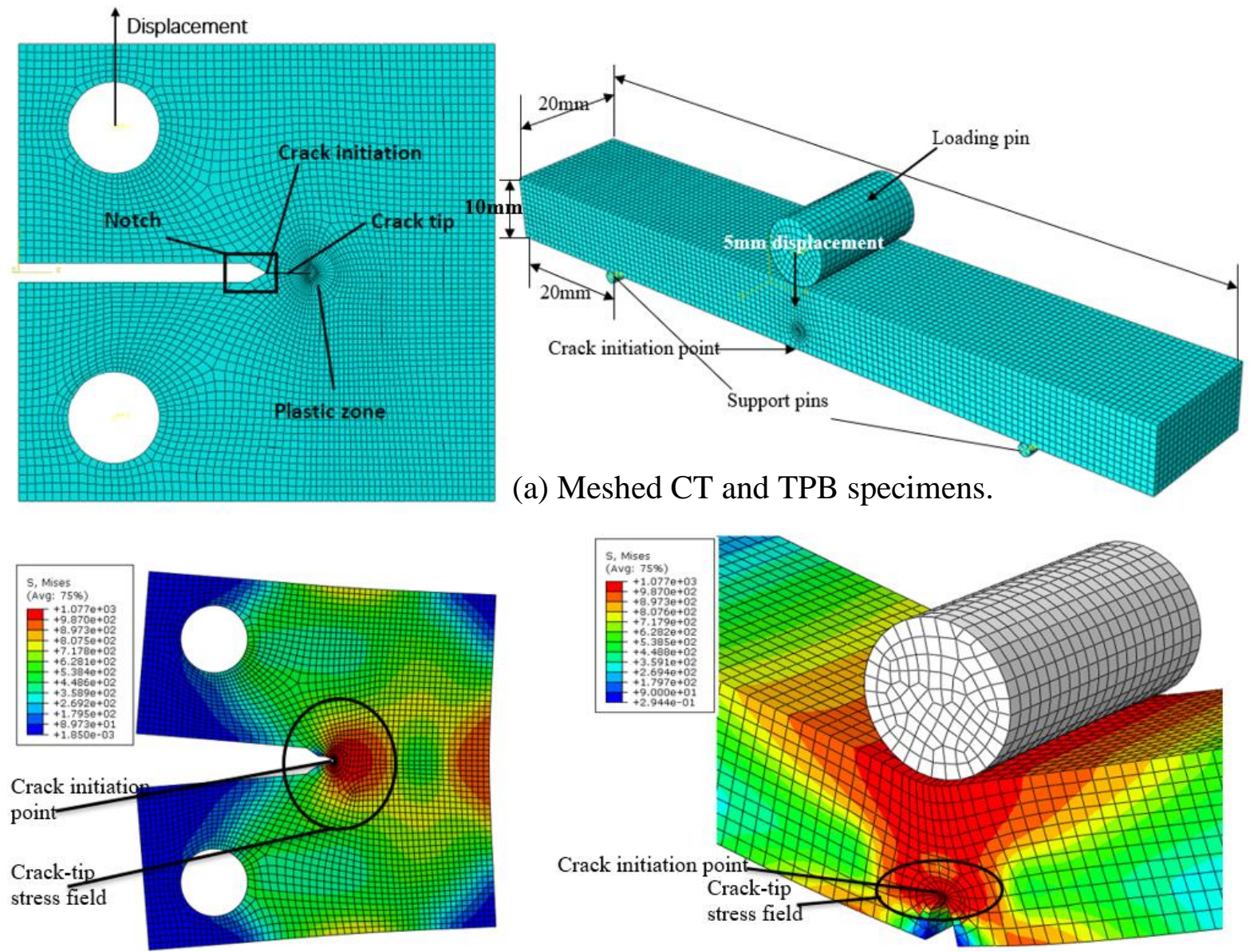

(b) Stress levels for deformed CT and TPB full-scale titanium models.

Figure 12. CT and TPB specimens used in finite similitude analysis.

Similarly, a three-point bending model specimen consisting of a beam with dimensions $10 \mathrm{~mm}$ height, $120 \mathrm{~mm}$ length (between loading points) and $20 \mathrm{~mm}$ depth is depicted in Fig. 12(a) along with the Abaqus model highlighting the mesh used in the analysis. In this case the loading pin is displaced $5 \mathrm{~mm}$ to induce plastic behaviour, and 8-noded continuum 3D stress elements (C3D8R) form the mesh. The results of the Abaqus simulation for the CT and TPB specimens for titanium are depicted in Fig. 12(b), where not unexpectedly high stress levels are apparent at the crack tip.

\subsection{Virtual material models}

The finite similitude theory does not require the formulation of constitutive models as the stress and strain fields are directly provided by Eqs. (28). It is of interest nonetheless in view of the uniaxial data in Fig. 11 and the ease at which Abaqus accepts discrete data to form a multi-axial constitutive law based on equivalent stress and strain. Incorporation of the data in Fig. 11 and the running of Abaqus readily facilitates the determination of J-integrals. In the 
case of the CT and TPB specimens depicted in Fig. 12, fifteen contours are located around each of the plastic zones and the results on the averaging fourteen of these are provided in Table 9 for both zeroth and first-order material models. It is evident on examination of the table that first-order provides a significant improvement on the zeroth-order predictions. Each model is aiming to capture the full-scale J-integral values for titanium (i.e. CT (570.4 $\left.\mathrm{kJ} / \mathrm{m}^{2}\right)$, TPB $\left(746.9 \mathrm{~kJ} / \mathrm{m}^{2}\right)$ ) and as revealed in the table the virtual material Ti-S-C provides the closest result.

Table 9. Results of numerical simulations of J-integral.

\begin{tabular}{|c|c|c|c|c|c|}
\hline & Material & $\begin{array}{l}\text { J-integral for } \\
\mathrm{CT}\left(\mathrm{kJ} / \mathrm{m}^{2}\right)\end{array}$ & $\begin{array}{l}\text { Error in CT } \\
\text { test }\end{array}$ & $\begin{array}{l}\text { J-integral for } \\
\text { TPB }\left(\mathrm{kJ} / \mathrm{m}^{2}\right)\end{array}$ & $\begin{array}{c}\text { Error in TPB } \\
\text { test }\end{array}$ \\
\hline Physical (real) & Titanium & 570.4 & - & 746.9 & - \\
\hline \multirow{5}{*}{$\begin{array}{l}\text { Zeroth order } \\
\text { (virtual) }\end{array}$} & Ti-SS & 584.4 & $2.5 \%$ & 824.2 & $10.3 \%$ \\
\hline & Ti-S & 606.2 & $6.3 \%$ & 780.8 & $4.5 \%$ \\
\hline & Ti-C & 714.4 & $25.2 \%$ & 952.8 & $27.6 \%$ \\
\hline & Ti-Tung & 533.3 & $6.5 \%$ & 693.8 & $7.1 \%$ \\
\hline & Ti-Al & 758.1 & $32.9 \%$ & 994.3 & $33.1 \%$ \\
\hline \multirow{6}{*}{$\begin{array}{l}\text { First order } \\
\text { (virtual) }\end{array}$} & Ti-SS-S & 547.0 & $4.1 \%$ & 700.8 & $6.2 \%$ \\
\hline & Ti-SS-C & 548.7 & $3.8 \%$ & 701.2 & $6.1 \%$ \\
\hline & Ti-SS-Tung & 548.0 & $3.9 \%$ & 698.4 & $6.5 \%$ \\
\hline & Ti-S-C & 574.4 & $0.7 \%$ & 734.6 & $1.6 \%$ \\
\hline & Ti-C-Tung & 556.2 & $2.5 \%$ & 695.7 & $6.9 \%$ \\
\hline & Ti-SS-Al & 549.0 & $3.8 \%$ & 701.3 & $6.1 \%$ \\
\hline
\end{tabular}

Examination of Table 9 reveals a similar trend to those shown in Figs. 10 and 11 and not too unexpectedly best results coming from those material combinations that provide the closest match to the stress-strain curve for titanium. Overall, the results of the study have confirmed the improvement that can be achieved by the adding of an additional trial-space experiment.

\subsection{Direct application of similitude identities}


As alluded above the similitude theory can lift experimental, analytical and numerical results directly and does not require the formulation of a constitutive model. Focus in this section, is on the normal stress in the y-direction (i.e. $\sigma_{p s}^{22}$ ) and the applications of finite similitude identities for stress Eq. (14c) and displacement Eq. (14e). The analysis is performed on the CT specimen, with the trial-models 1 and 2 being half and quarter size of physical model and subjected to displacements $1 \mathrm{~mm}$ and $0.5 \mathrm{~mm}$, respectively. The trial models are effectively scaled replicas of the full-scale model depicted in Fig. 12 with the exact same number of elements and element type. The $\sigma_{p s}^{22}$ stress contour for titanium in the physical space is depicted in Fig. 13 and the objective here is to ascertain how close a virtual model constructed from the results of steel and stainless trial models replicates this in the zone near the crack tip.

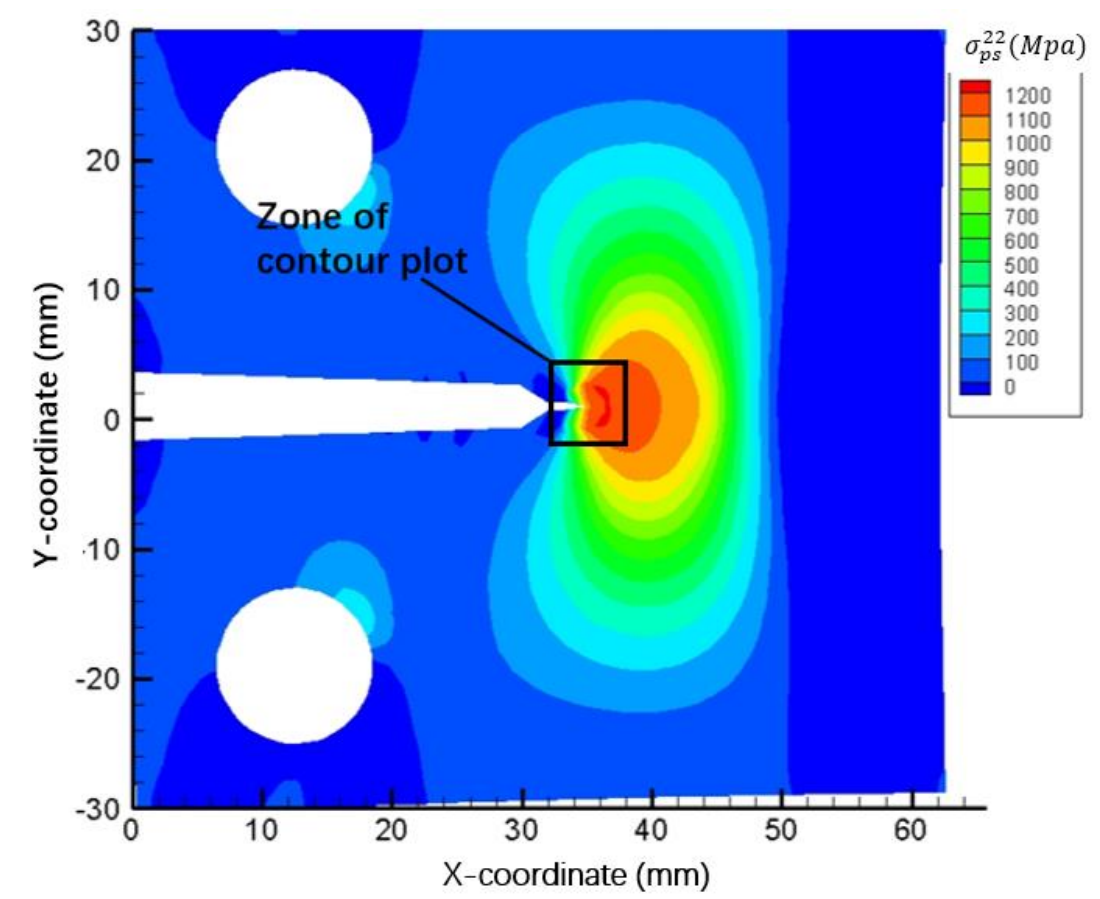

Figure 13. Contour plot of stress for the physical-space CT specimen (titanium).

The results of the study are presented in Fig. 14 drawn using Tecplot (360 EX 2019 R1) and based on output from Abaqus (real models in the figure) with the virtual stress field obtained from Eq. (14c). The results provide a good illustration of the direct application of the finite similitude scaling identities without the need for a constitutive model and consequently an approach ideal for directly relating experimental results. 


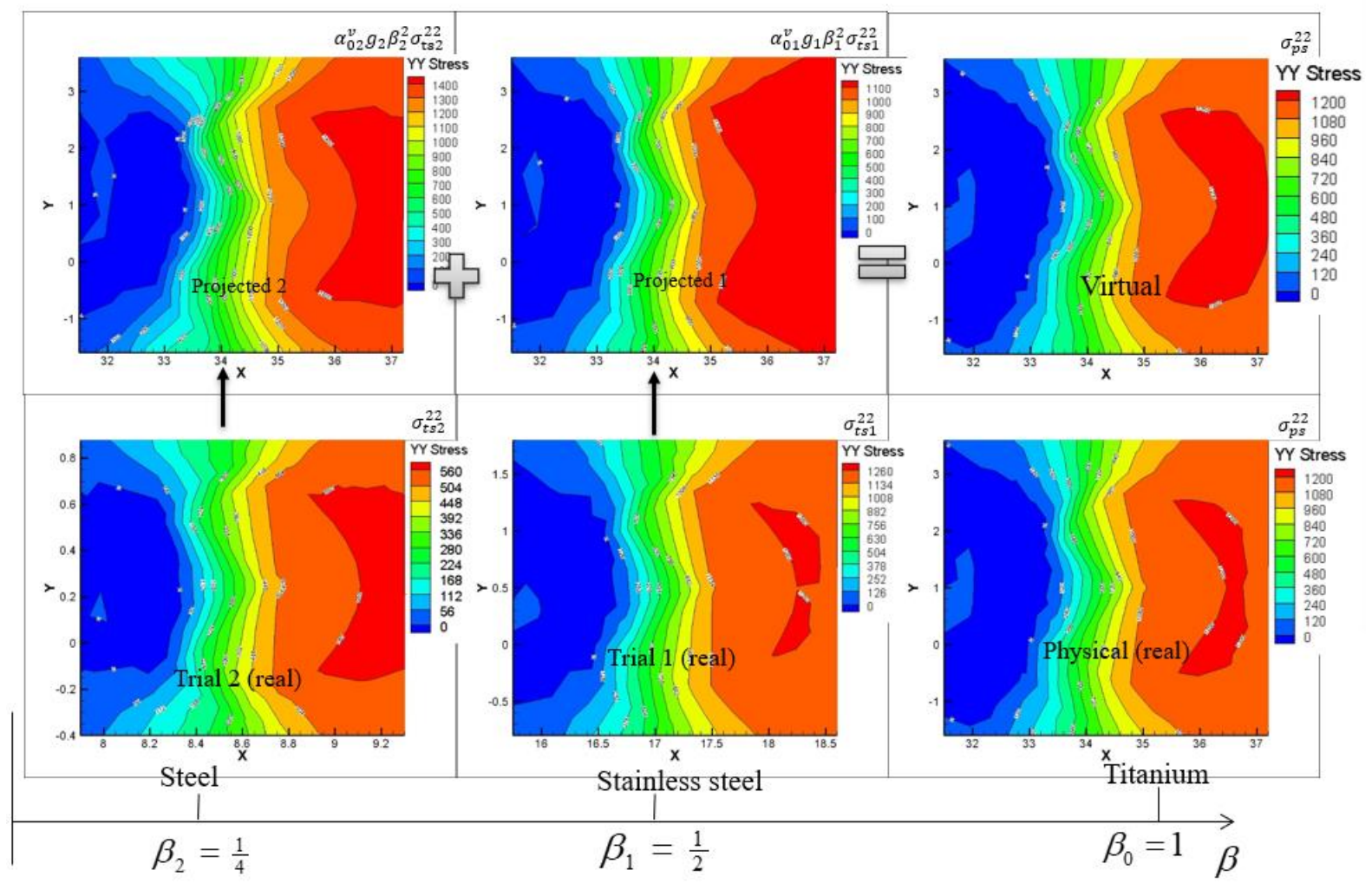

Figure 14. Virtual contour plot produced by trial data (stainless steel and steel).

Although a constitutive model is not required it is of interest to contrast the direct and virtualmaterial model approaches and the results for this are presented in Fig. 15, where reasonably consistent results are revealed.
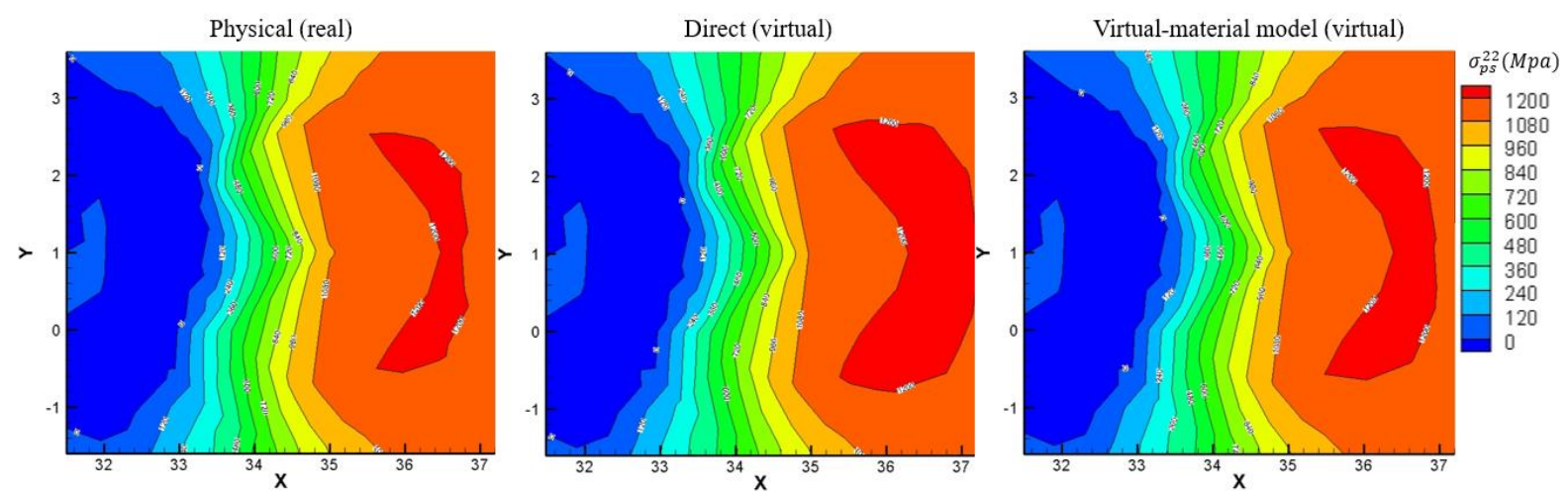

Figure 15. Contour for real, direct and virtual-material models.

\section{A practical demonstration in Ansys}

To demonstrate that the approach presented has practicality and additionally is not reliant on a particular package a semi-elliptical crack located on the outer wall of a cylindrical pressure vessel is examined in this section using the commercial software package ANSYS [43]. The problem is slightly more nuanced than those described above being truly $3 \mathrm{D}$ in nature with 
focus on calculating stress intensity factors (SIFs) and J-integrals. The details of the crack and the pressure vessel are presented in Fig.16 along with the finite element mesh employed. The pressure vessel is pressurised internally but constrained laterally to impose plane strain conditions on the crack. The cross-section view of the crack presented Fig. 16(b) shows crack depth (minor radius) $b$, half crack length (major radius) $a$, and thickness $T$ of the cylindrical pressure vessel. The pressure vessel dimensions are outer radius $R_{O}=1.5 \mathrm{~m}$, inner radius $R_{i}=1.4 \mathrm{~m}$, length $L=8 \mathrm{~m}$ and the following ratios apply: $\frac{R_{i}}{T}=14, \frac{b}{T}=\frac{b}{a}=$ 0.5 .

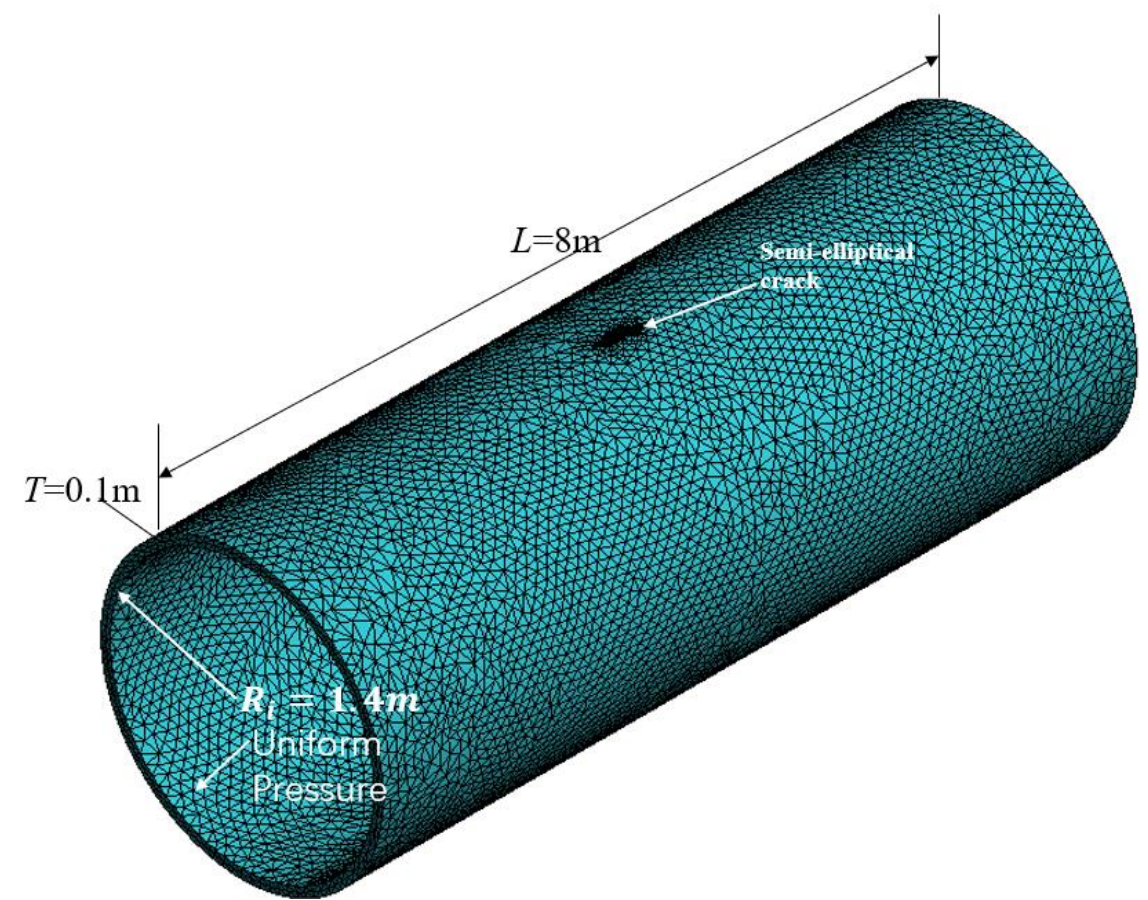

(a) Mesh and overall dimensions
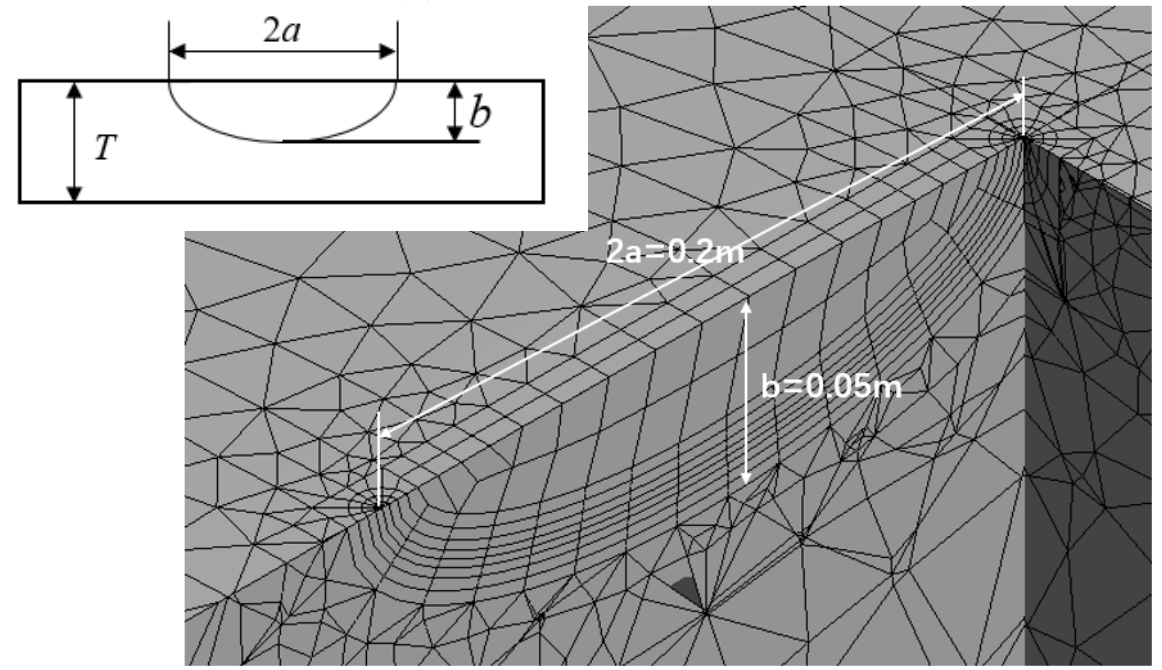

(b) Cross section of the semi-elliptical crack

Figure 16. Mesh and crack details for an internally pressurized pressure vessel 
The FE mesh for the pressure vessel depicted in Fig. 16(a) is made up of 52283 solid tetrahedron elements (element size $\sim 0.1 \mathrm{~m}$ ) and a total of 100234 nodes. The overall dimensions of the semi-elliptical (SE) crack are provided in Fig. 16(b) and conform to the relationship $\frac{b}{a}=0.5$. The ANSYS software evaluates SIFs using a contour integration procedure with the facility to set the number contours. In this study the number of contours around the crack tip is set equal to be 3 with the largest contour radius equalling $0.05 \mathrm{~m}$.

\subsection{Linear elastic analysis}

Zeroth-order theory is sufficient for a linear-elastic analysis and since the SIF $K \propto \sigma \sqrt{\pi a}$ and $J=\frac{K^{2}}{E^{\prime}}$ the analysis in Section 6.1 applies, which for an identical material (i.e. titanium) at full size and scale, Eq. (24) provides $\alpha_{01}^{v} g_{1}=\frac{1}{\beta_{1}^{2}} \frac{E_{p s}^{\prime}}{E_{t s 1}^{\prime}}=\frac{1}{\beta_{1}^{2}}$. The precise relationship for the SIF for the SE crack is

$$
K=\sigma \sqrt{\pi a} \cdot f\left(a^{\prime}\right)
$$

where $f\left(a^{\prime}\right)$ is a shape function and takes the form,

$$
f\left(a^{\prime}\right)=\sqrt{1+0.52 a+1.29 a^{2}-0.07 a^{3}}
$$

and where $a^{\prime}=\frac{a}{\sqrt{R_{i} T}}$, which is a dimensionless parameter unaffected by scale and consequently $a_{p s}^{\prime}=a_{t s}^{\prime}$.

It follows therefore that $\boldsymbol{\sigma}_{p s}=\alpha_{01}^{v} g_{1} \beta_{1}^{2} \boldsymbol{\sigma}_{t s 1}=\boldsymbol{\sigma}_{t s 1}, K_{p s}=\alpha_{01}^{v} g_{1} \beta_{1}^{3 / 2} K_{t s 1}=\beta_{1}^{-1 / 2} K_{t s 1}$ and $J_{p s}=\alpha_{01}^{v} g_{1} \beta_{1} J_{t s 1}=\beta_{1}^{-1} J_{t s 1}$, which are relationships that are confirmed on comparison of virtual and real results presented Fig. 17 (i.e. Ti-Ti and Ti curves), with 5MPa internal uniform pressure and $\beta_{1}=\frac{1}{2}$ and $\frac{1}{4}$. Note that the measure used along the abscissas in Fig. 17(b) and (c) is the length along the middle contour (of the 3 contours) starting from point 1 in Fig 17(a) and ending at point 2. In the situation where a different material is used at scale, then Eq. (24) provides $\alpha_{01}^{v} g_{1}=\frac{1}{\beta_{1}^{2}} \frac{E_{p s}^{\prime}}{E_{t s 1}^{\prime}}$ and consequently $\boldsymbol{\sigma}_{p s}=\alpha_{01}^{v} g_{1} \beta_{1}^{2} \boldsymbol{\sigma}_{t s 1}=\frac{E_{p s}^{\prime}}{E_{t s 1}^{\prime}} \boldsymbol{\sigma}_{t s 1}$, which means the pressure in the scaled model must change as shown in Table. 10 for a selection of materials. Note also in this case $K_{p s}=\alpha_{01}^{v} g_{1} \beta_{1}^{3 / 2} K_{t s 1}=\frac{E_{p s}^{\prime}}{E_{t s 1}^{\prime}} \beta_{1}^{-1 / 2} K_{t s 1}$ and $J_{p s}=\alpha_{01}^{v} g_{1} \beta_{1} J_{t s 1}=\frac{E_{p s}^{\prime}}{E_{t s 1}^{\prime}} \beta_{1}^{-1} J_{t s 1}$, which are confirmed to reasonable accuracy by the results presented in Fig. 17. 
Table 10. Applied pressure for scaled material models

\begin{tabular}{|l|l|l|l|l|l|}
\hline Materials & Titanium & Stainless steel & Steel & Copper & Aluminium \\
\hline $\begin{array}{l}\text { Young's modulus } \\
(\mathrm{GPa})\end{array}$ & 120 & 200 & 190 & 130 & 70 \\
\hline Pressure (MPa) & 5 & 8.3333 & 7.9167 & 5.4167 & 2.9167 \\
\hline
\end{tabular}

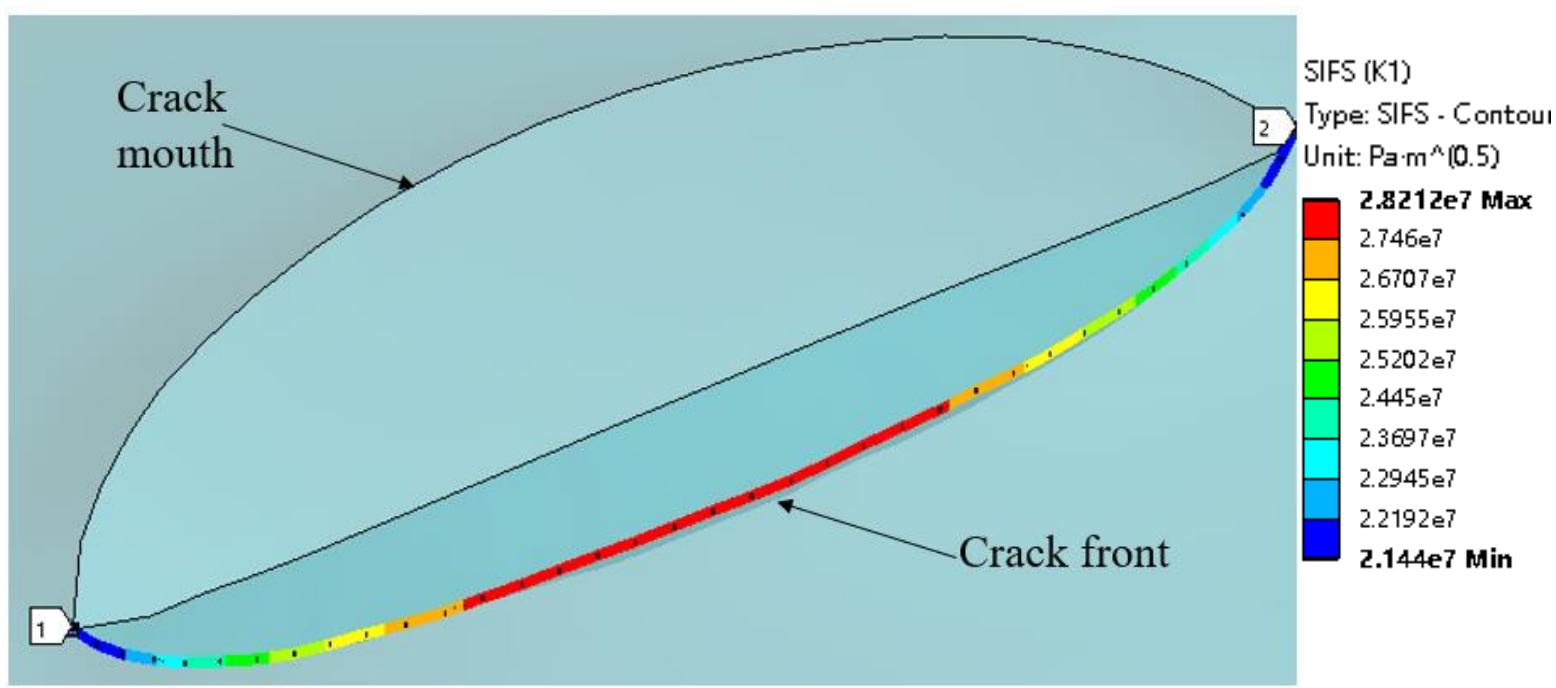

(a) Contour at crack front used by ANSYS

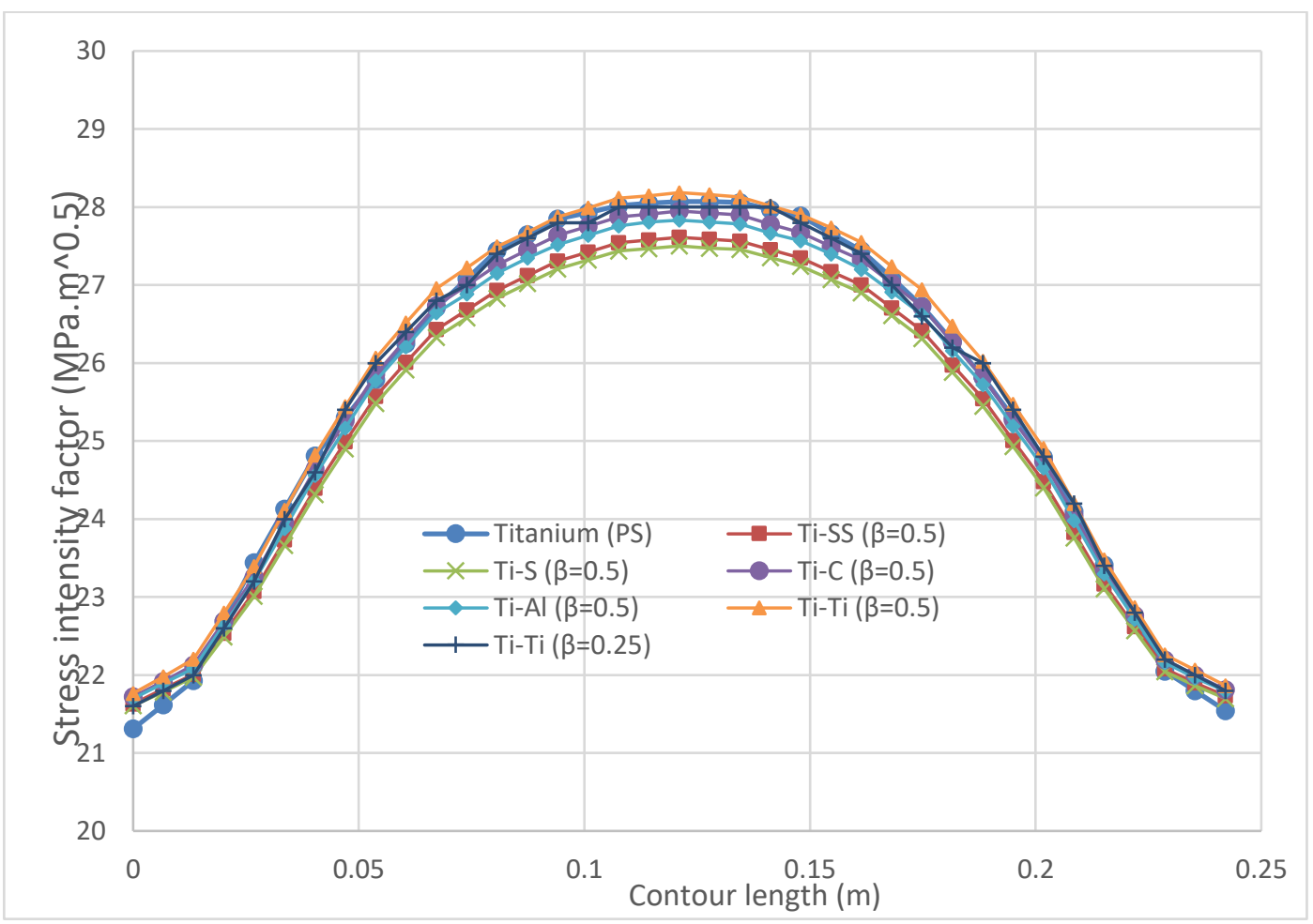

(b) SIFs along contour 


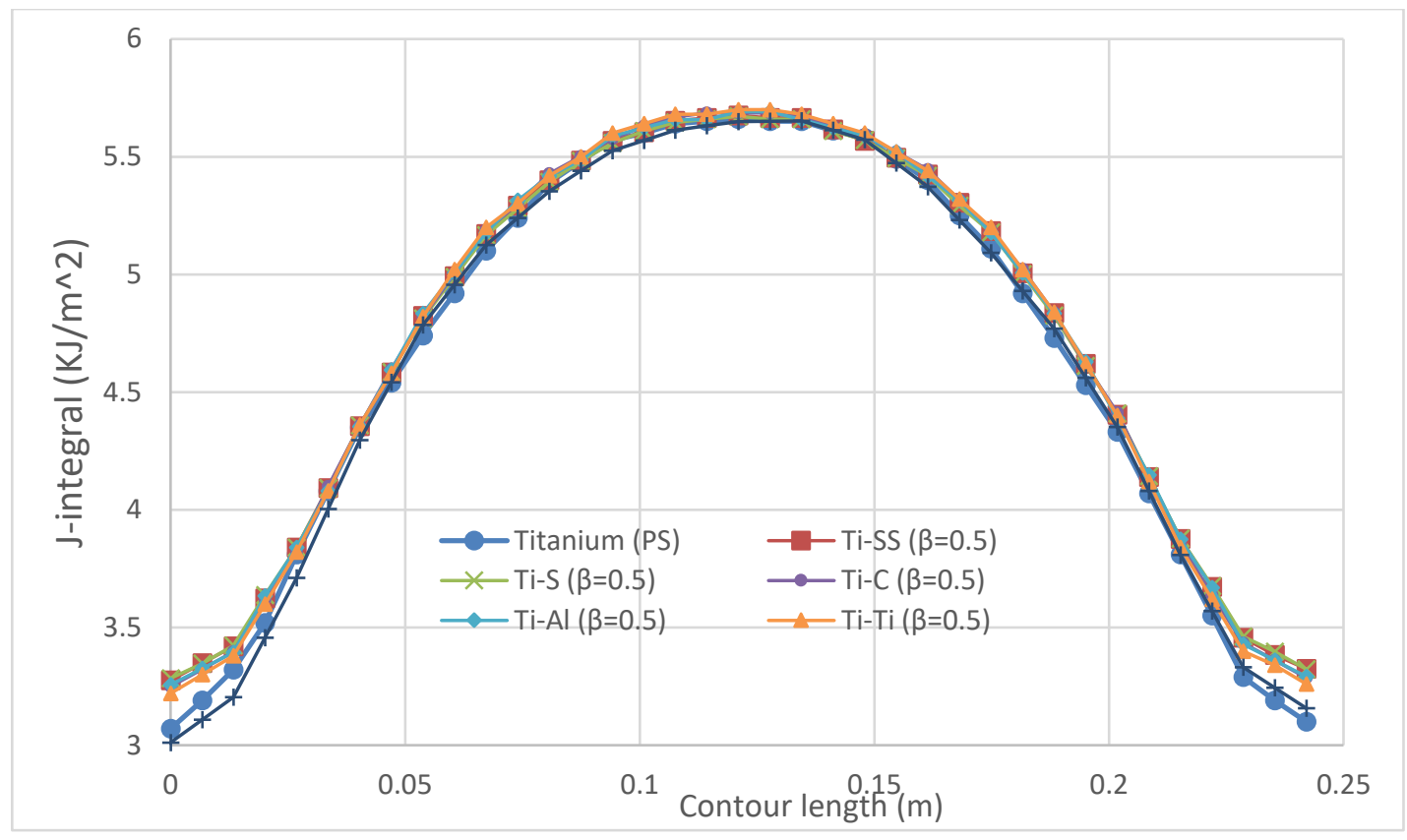

(c) J-integral along contour

Figure 17. J-integrals and SIFs along contour shown in (a)

\subsection{Plastic analysis}

Things are slightly more involved in the situation where the pressure loading is sufficient to initiate plastic behaviour. The approach outlined in Section 7 is applied here with the targeting of yield stress for zeroth order and both yield and mean stress (i.e. Eq. (31)) first order. The SIF in this situation can again be estimated using Eq. (33) but requires a plastic correction, according to Marie and Nédélec [44], which for a pressure vessel takes the form

$$
K_{p l}=K_{e l}\left[1+\frac{1}{2\left(1-v^{2}\right)} \sqrt{1-\left(\frac{1}{1+2 \frac{r_{y}}{a}}\right)^{2}}\right] \sqrt{\frac{\frac{a}{2}+r_{y}}{\frac{a}{2}}}
$$

where $r_{y}$ is a measure of the extent of the plastic zone at the crack tip, and one possible estimate is Irwin's formula $r_{y}=\frac{1}{6 \pi}\left(\frac{K_{e l}}{Y}\right)^{2}$ with yield stress $Y$.

The presence of $K_{e l}$ in $r_{y}$ means that $K_{p l}$ is nonlinearly related to $K_{e l}$ and hence to the shape function and the ratio between applied stress and yield stress (see Shlyannikov [45]). The applied stress between physical and trial space still obeys $\boldsymbol{\sigma}_{p s}=\alpha_{01}^{v} g_{1} \beta_{1}^{2} \boldsymbol{\sigma}_{t s}$, thus, in order to negate differences in plastic correction at scale it is necessary to set $\alpha_{01}^{v} g_{1} \beta_{1}^{2}=\frac{Y_{p s}}{Y_{t s 1}}$. This again provides different pressures at scale for different materials as tabulated in Table 11. 
Table 11. Applied pressure for each material in plastic model

\begin{tabular}{|l|l|l|l|l|l|}
\hline Materials & Titanium & Stainless steel & Steel & Copper & Aluminium \\
\hline Yield stress (MPa) & 812 & 881 & 323.5 & 92.2 & 98.4 \\
\hline Pressure (MPa) & 50 & 54.25 & 19.99 & 5.68 & 6.06 \\
\hline
\end{tabular}

Note that the relationship between stress intensity factors for first-order theory is

$$
K_{p s}=\alpha_{01}^{v} g_{1} \beta_{1}^{3 / 2} K_{t s 1}+R_{1}\left(\alpha_{01}^{v} g_{1} \beta_{1}^{\frac{3}{2}} K_{t s 1}-\alpha_{02}^{v} g_{2} \beta_{2}^{\frac{3}{2}} K_{t s 2}\right)
$$

with zeroth order returned on setting $R_{1}=0$.

The results of the trial are provided in Fig. 18 with reasonable accuracy returned for both zeroth and first order except for Ti-Al in Fig. 18(a). Finally, Fig. 19 highlights the vast improvement possible for J-integrals using the first order theory with the J-integrals determined using the in-built facility in ANSYS. The accuracies visible in the figures reflect to a large extent the closeness of the curves to titanium in Figs. 10 and 11.

(a)

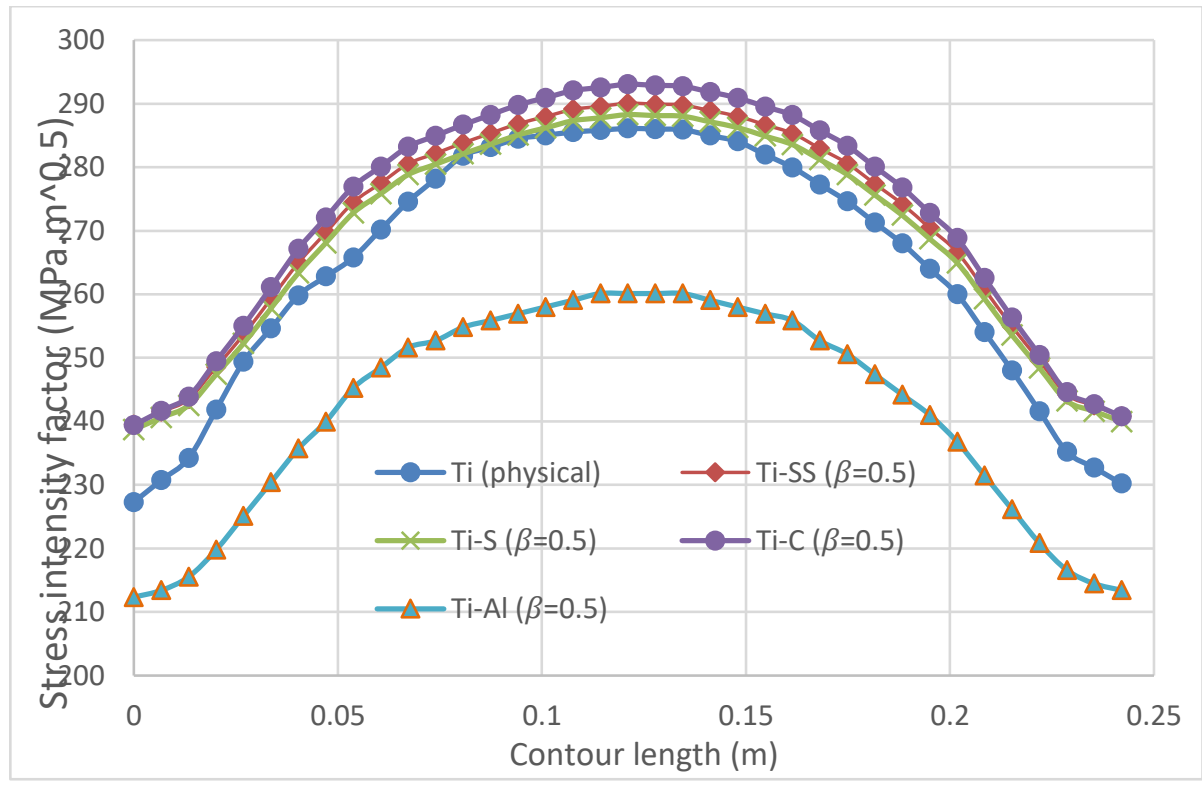


(b)

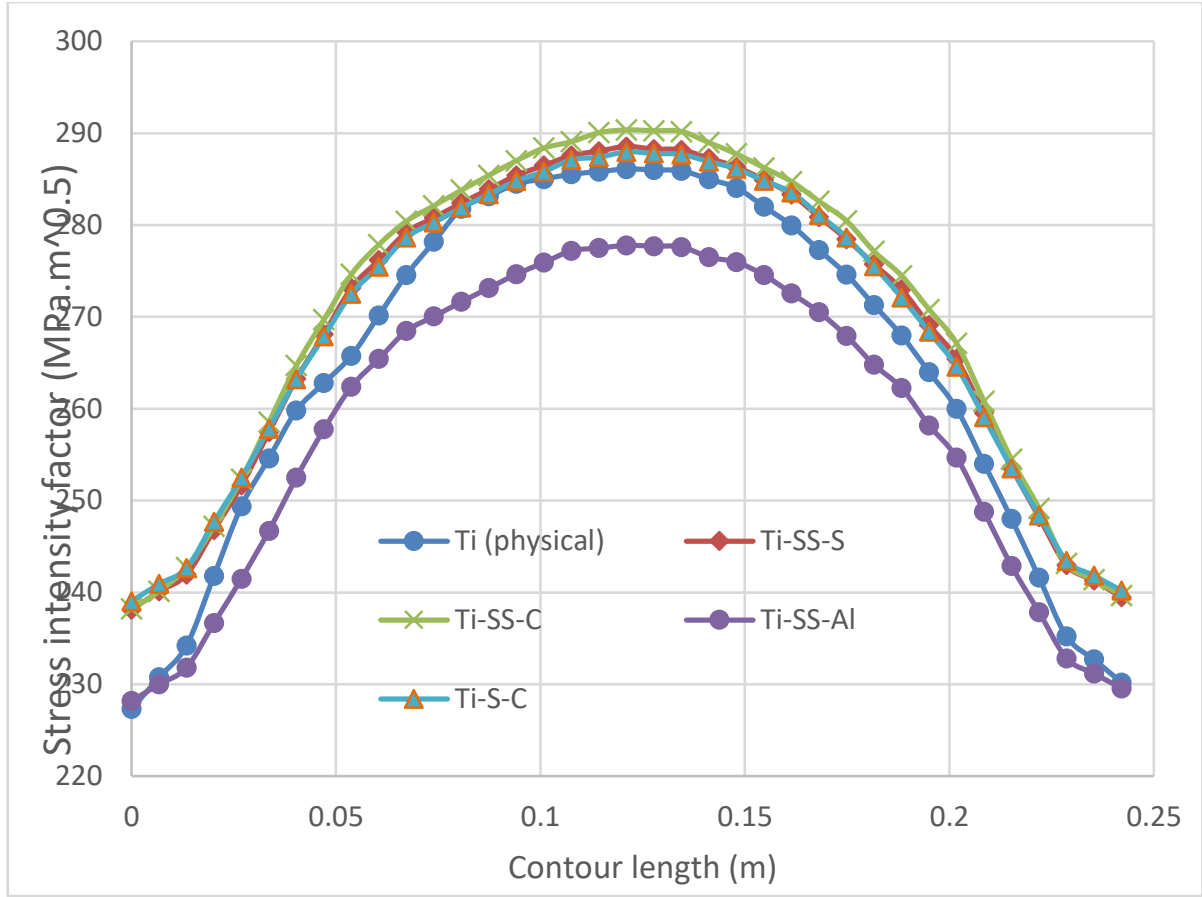

Figure 18. SIF predictions with (a) zeroth-order and (b) first-order using the in-built facility in ANSYS and Eq. (36).

(a)

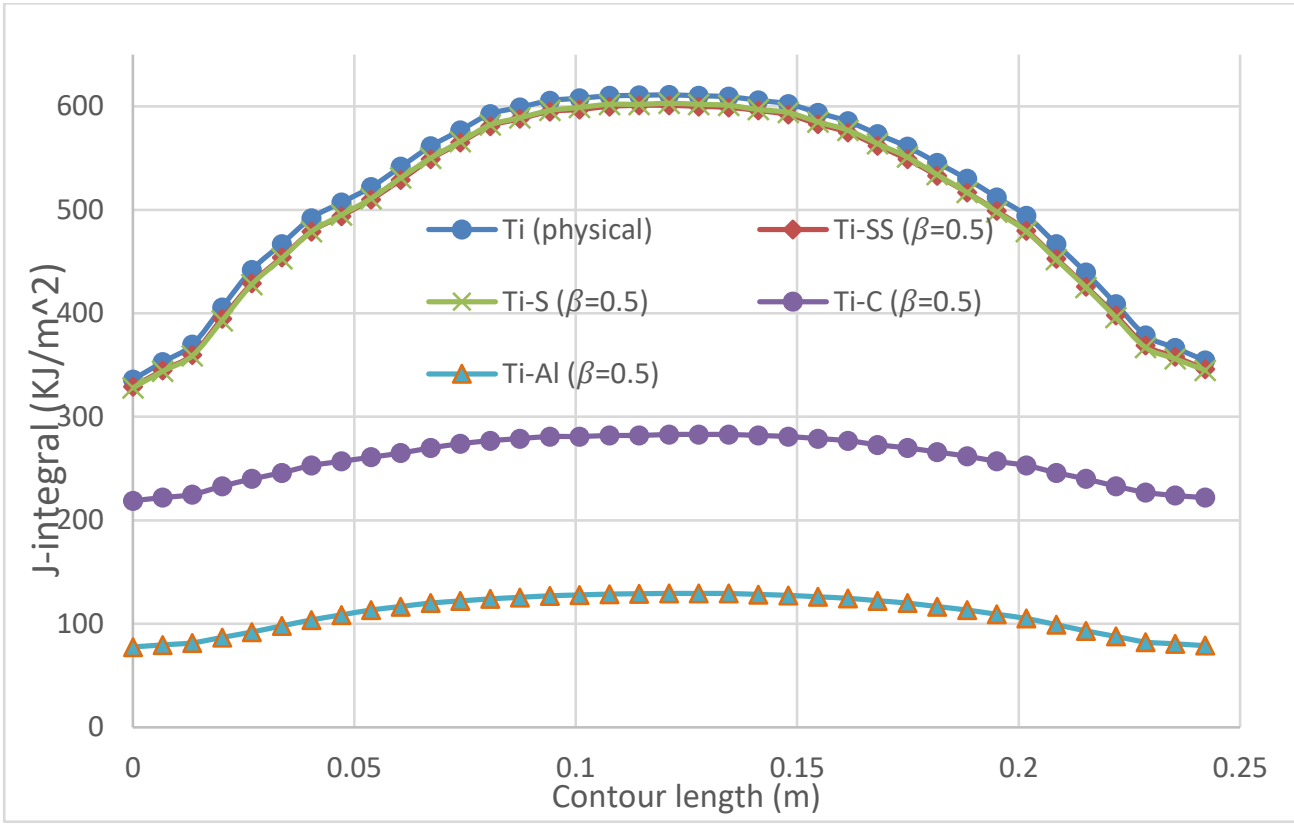


(b)

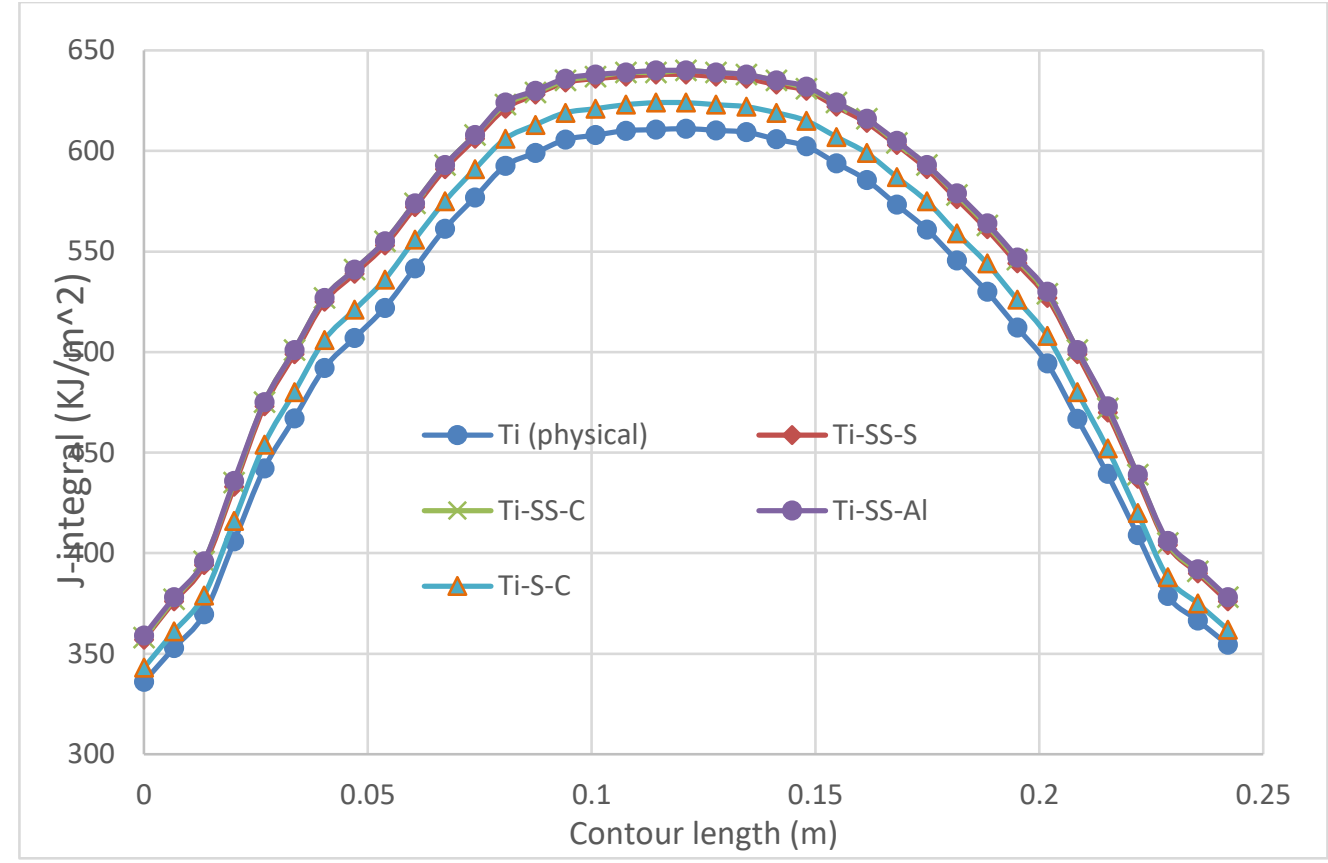

Figure 19. J-integral predictions for (a) zeroth-order and (b) first-order using the in-built facility within ANSYS.

\section{Conclusion}

The paper examines a new scaling theory for the creation of physical models for the representation of full-scale cracked specimens. A particularly novel feature is the employment of two scaled fracture-mechanics experiments at distinct scales and the application of a theory founded on the metaphysical concept of space scaling. The following conclusions can be drawn from the investigation outlined in the paper:

1. The finite similitude theory has been further developed so that it captures all scale dependencies that arise in the fields describing fracture mechanics.

2. A new differential form of similarity has been established, which when integrated links information across two scaled-fracture experiments to the full-scale behaviour.

3. The new theory has been shown to be equally applicable to analytical and numerical fracture models and provides improvements in accuracy, which on occasions can be markedly superior to those obtained from a single-scale trial experiment.

4. Scale effects as previously defined by dimensional analysis can up to a limited extent be accommodated and cease to be scale effects in the new theory (e.g. proportional field differences are now possible).

5. The efficient determination of scaling parameters has been shown possible by the application of a proportional-fields assumption. 
More specifically for the trial experiments performed it has been shown that:

1. Linear elastic fracture mechanics was captured exactly for the CT specimen by allowing the length scale for the trial experiments to be determined as part of the analysis.

2. Zeroth-order theory involving one single trial experiment proved to be insufficient for elastoplastic fracture mechanics, but significant improvement was shown possible with two trial scale experiments.

3. Contour integral fracture mechanics was shown to be possible and greatest accuracy was returned with the involvement of two trial-scale experiments.

\section{ACKNOWLEDGEMENTS}

The authors would like to acknowledge the parents of Mr Jingwen Zhang for providing support to facilitate his doctoral research at the University of Manchester. 


\section{References}

1. Atkins, A.G. (1999). Scaling laws for elastoplastic fracture. Fracture Scaling, pp.51-65.

2. Bažant, Z.P. and Chen, E.-P. (1997). Scaling of Structural Failure. Applied Mechanics Reviews, 50(10), pp.593-627.

3. Drazetic, P., Ravalard, Y., Dacheux, F. and Marguet, B. (1994). Applying non-direct similitude technique to the dynamic bending collapse of rectangular section tubes. International Journal of Impact Engineering, 15(6), pp.797-814.

4. Carpinteri, A., Paggi, M., Daniewicz, S.R. and Dean, S.W. (2011). Dimensional Analysis and Fractal Modeling of Fatigue Crack Growth. Journal of ASTM International, 8(10), p.104105.

5. Mazzariol, L.M., Oshiro, R.E. and Alves, M. (2016). A method to represent impacted structures using scaled models made of different materials. International Journal of Impact Engineering, 90, pp.81-94.

6. Alves, M. and Oshiro, R.E. (2006). Scaling the impact of a mass on a structure. International Journal of Impact Engineering, 32(7), pp.1158-1173.

7. Mazzariol, L.M. and Alves, M. (2019). Similarity laws of structures under impact load: Geometric and material distortion. International Journal of Mechanical Sciences, 157158, pp.633-647.

8. Mazzariol, L.M. and Alves, M. (2019). Experimental verification of similarity laws for impacted structures made of different materials. International Journal of Impact Engineering, 133, p.103364.

9. Neuberger, A., Peles, S. and Rittel, D. (2007). Scaling the response of circular plates subjected to large and close-range spherical explosions. Part I: Air-blast loading. International Journal of Impact Engineering, 34(5), pp.859-873.

10. Neuberger, A., Peles, S. and Rittel, D. (2007). Scaling the response of circular plates subjected to large and close-range spherical explosions. Part II: Buried charges. International Journal of Impact Engineering, 34(5), pp.874-882.

11. Grossmann, S. and Lohse, D. (2000). Scaling in thermal convection: a unifying theory. Journal of Fluid Mechanics, [online] 407, pp.27-56. Available at: https://research.utwente.nl/en/publications/scaling-in-thermal-convection-a-unifying-view [Accessed 20 Apr. 2020].

12. Bažant, Z.P. and Novák. D. (2000). Energetic-Statistical Size Effect in Quasibrittle Failure at Crack Initiation. ACI Materials Journal, 97(3).

13. Kneller, B. and Buckee, C. (2000). The structure and fluid mechanics of turbidity currents: a review of some recent studies and their geological implications. Sedimentology, 47, pp.62-94.

14. Mohamed, M.S., Foster, A.D., Lin, J., Balint, D.S. and Dean, T.A. (2012). Investigation of deformation and failure features in hot stamping of AA6082: Experimentation and modelling. International Journal of Machine Tools and Manufacture 53(1), pp. 27-38.

15. Rayleigh, L. (1915). The Principle of Similitude. Nature, 95(2373), pp.202-203.

16. Goodier, J.N. and Thomson, W.T. (1944). Applicability of Similarity Principles to Structural Models.ntrs.nasa.gov. [online] Available at: https://ntrs.nasa.gov/search.jsp?R=19930081676 [Accessed 20 Apr. 2020].

17. Goodier. J.N. (1950). Dimensional Analysis. Wiley, New York.

18. Simitses, G.J. and Rezaeepazhand, J. (1995). Structural Similitude and Scaling Laws for Buckling of Cross-Ply Laminated Plates. Journal of Thermoplastic Composite Materials, 8(3), pp.240-251.

19. Cheng, Y.-T. and Cheng, C.-M. (2004). Scaling, dimensional analysis, and indentation measurements. Materials Science and Engineering: R: Reports, 44(4-5), pp.91-149. 
20. Brinell. J.A. (1901) Congres International des $\mathrm{M}$ des de Construction (Paris), tome 2, 8394.

21. Bažant, Z.P. (1984). Size Effect in Blunt Fracture: Concrete, Rock, Metal. Journal of Engineering Mechanics, 110(4), pp.518-535.

22. Kirane, K. and Bažant, Z.P. (2016). Size effect in Paris law and fatigue lifetimes for quasibrittle materials: Modified theory, experiments and micro-modeling. International Journal of Fatigue, 83, pp.209-220.

23. Carpinteri, A., Paggi, M., Daniewicz, S.R. and Dean, S.W. (2011). Dimensional Analysis and Fractal Modeling of Fatigue Crack Growth. Journal of ASTM International, 8(10), p.104105.

24. Le, J.-L., Manning, J. and Labuz, J.F. (2014). Scaling of fatigue crack growth in rock. International Journal of Rock Mechanics and Mining Sciences, 72, pp.71-79.

25. Ray, S. and Chandra Kishen, J.M. (2011). Fatigue crack propagation model and size effect in concrete using dimensional analysis. Mechanics of Materials, 43(2), pp.75-86.

26. Davey K, Darvizeh R, Al-Tamimi A. (2017). Scaled Metal Forming Experiments: A Transport Equation Approach. International Journal of Solids and Structures, 125, pp. 184-205.

27. Sadeghi, H., Davey, K., Darvizeh, R. and Darvizeh, A. (2019). A scaled framework for strain rate sensitive structures subjected to high rate impact loading. International Journal of Impact Engineering, 125, pp.229-245.

28. Sadeghi, H., Davey, K., Darvizeh, R. and Darvizeh, A. (2019). Scaled models for failure under impact loading. International Journal of Impact Engineering, 129, pp.36-56.

29. Davey, K. and Darvizeh, R. (2016). Neglected transport equations: extended RankineHugoniot conditions and $\mathrm{J}$-integrals for fracture. Continuum Mechanics and Thermodynamics, 28(5), pp.1525-1552.

30. Gurtin, M.E. (1995). The nature of configurational forces. Archive for Rational Mechanics and Analysis, 131(1), pp.67-100.

31. Gewin, V. (2008). Shock physics. Nature, 454(7200), pp.134-134.

32. Al-Tamimi, A., Darvizeh, R. and Davey, K. (2018). Experimental investigation into finite similitude for metal forming processes. Journal of Materials Processing Technology, 262, pp.622-637.

33. Ochoa-Cabrero, R., Alonso-Rasgado, T. and Davey, K. (2018). Scaling in biomechanical experimentation: a finite similitude approach. Journal of The Royal Society Interface, 15(143), p.20180254.

34. Moghaddam, M., Darvizeh, R., Davey, K. and Darvizeh, A. (2018). Scaling of the powder compaction process. International Journal of Solids and Structures, 144-145, pp.192-212.

35. Davey, K., Sadeghi, H., Darvizeh, R., Golbaf, A. and Darvizeh, A. (2021). A Finite Similitude Approach to Scaled Impact Mechanics. International Journal of Impact Engineering, 148, p.103744.

36. Rice, J.R. (1968). A Path Independent Integral and the Approximate Analysis of Strain Concentration by Notches and Cracks. Journal of Applied Mechanics, 35(2), pp.379-386.

37. Department of Defense. Military Standardization Handbook: Metallic Materials and Elements for Aerospace Vehicle Structures MIL-HDBK-5E, Volume 2. June 1987.

38. Holmquist, T.J. and Johnson, G.R. (2011). A Computational Constitutive Model for Glass Subjected to Large Strains, High Strain Rates and High Pressures. Journal of Applied Mechanics, 78(5).

39. Nicholas. T. (1980). Material behavior at high strain rates. Report AFWAL-TR-80-4053, USAF Wright Aeronautical Laboratories, Wright-Patterson Air Force Base, OH, USA. 
40. Antusch, S., Armstrong, D.E.J., Britton, T.B., Commin, L., Gibson, J.S.K.-L., Greuner, H., Hoffmann, J., Knabl, W., Pintsuk, G., Rieth, M., Roberts, S.G. and Weingaertner, T. (2015). Mechanical and microstructural investigations of tungsten and doped tungsten materials produced via powder injection molding. Nuclear Materials and Energy, 3-4, pp.22-31.

41. Salih, S., Davey, K. and Zou, Z. (2016). Rate-dependent elastic and elasto-plastic cohesive zone models for dynamic crack propagation. International Journal of Solids and Structures, 90, pp.95-115.

42. ABAQUS 6.14, Dassault Systèmes (Providence, RI, USA)

43. ANSYS, Inc, (2019) ANSYS Users' manual, Swanson Analysis System.

44. Marie, S., and Nédélec, M. (2007). A New Plastic Correction for the Stress Intensity Factor of an Under-Clad Defect in a PWR Vessel Subjected to a Pressurised Thermal Shock. Int. J. Pressure Vessels Piping, 84(3), pp. 159-17

45. Shlyannikov, V. (2016). Nonlinear stress intensity factors in fracture mechanics and their applications. Procedia Structural Integrity, 2, pp.744-752. 


\section{Nomenclature}

$\alpha_{0}^{\Psi} \quad$ Scalar for transport equation for field $\Psi$

$\alpha^{\rho} \quad$ Scalar for continuity equation

$\alpha^{v} \quad$ Scalar for momentum equation

$\alpha^{u} \quad$ Scalar for movement equation

$\alpha^{\omega} \quad$ Scalar for energy

$\alpha_{0}^{\Psi} T_{0}^{\Psi} \quad$ Scaled transport equation for field $\Psi$

$a \quad$ Crack length (or half crack length for semi-elliptical crack)

$A_{0} \quad$ Sectional area of the rod

$\beta \quad$ Length scalar

$\boldsymbol{b}_{p s}^{\Psi} \quad$ Source term in transport equation for field $\Psi$

$b \quad$ Crack depth of semi-elliptical crack

$\boldsymbol{e}_{i} \quad$ Directional vector for crack travel

E Young's modulus

F $\quad$ Space distortion matrix/tensor

$g \quad$ Time scalar

$G_{c} \quad$ Fracture energy per unit area (critical energy for fracture)

$J^{\Psi} \quad$ Flux term in transport equation for field $\Psi$

$J^{*} \quad$ Extended J-integral

$J_{c} \quad$ Critical J-integral at failure

$K_{I} \quad$ Stress intensity factor (Mode I crack)

$K_{I C} \quad$ Fracture toughness 


\begin{tabular}{|c|c|}
\hline$\ell$ & Length of rod \\
\hline $\boldsymbol{n}$ & Unit normal vector \\
\hline$R_{1}$ & First-order scaling parameter \\
\hline$R_{o}$ & Outer radius of the pressure vessel \\
\hline$R_{i}$ & Inner radius of the pressure vessel \\
\hline$t$ & Time \\
\hline$T$ & Thickness of the pressure vessel \\
\hline $\boldsymbol{\tau}$ & Surface traction vector \\
\hline$u$ & Displacement field \\
\hline $\mathrm{V}$ & Volume \\
\hline $\boldsymbol{v}^{*}$ & Velocity field for control volume motion \\
\hline$v$ & Poisson's ratio \\
\hline$x$ & Coordinate point \\
\hline$\chi$ & Coordinate point in reference control volume \\
\hline$Y$ & Yield stress \\
\hline$\rho$ & Material density \\
\hline $\boldsymbol{\sigma}$ & Stress field \\
\hline$\varepsilon$ & Strain field \\
\hline$\delta_{j}^{j}$ & Kronecker delta symbol \\
\hline$\delta_{c}$ & Critical separation in cohesive zone model \\
\hline$d \Gamma$ & Elemental surface-area vector \\
\hline$\Gamma^{*}$ & Orientable control volume boundary \\
\hline
\end{tabular}


Physical field

$\omega$

Strain-energy density

$\Omega^{*}$

Control volume 